\title{
On a Degenerate Quasilinear Elliptic Equation with Mixed Boundary Conditions
}

\author{
Kazuya HAYASIDA and Yasuhiko KAWAI \\ Kanazawa University and Intelligence Business School of Kanazawa \\ (Communicated by K. Kojima)
}

\section{Introduction}

Let $\Omega$ be a bounded simply connected domain in $\boldsymbol{R}^{n}$. The boundary $\partial \Omega$ is assumed to be of class $C^{1}$. Let $S$ be a compact $C^{1}$ manifold of dimension $n-2$ belonging to $\partial \Omega$. We assume that $S$ divide $\partial \Omega$ into two non-empty relatively open subsets $\partial_{1} \Omega$ and $\partial_{2} \Omega$, more precisely,

$$
\partial \Omega=\partial_{1} \Omega \cup \partial_{2} \Omega \cup S, \quad \partial_{1} \Omega \cap \partial_{2} \Omega=\varnothing .
$$

We assume that the usual function spaces $C^{k}(\bar{\Omega}), C_{0}^{k}(\Omega), L^{q}(\Omega), W^{1, q}(\Omega)$, $W_{0}^{1, q}(\Omega)$ are known. The norm in $W^{1, q}(\Omega)\left(L^{q}(\Omega)\right)$ is written with \|\|$_{1, q}$ $\left(\|\|_{q}\right)$, respectively. Throughout this paper let $2<p<\infty$, and let all functions be real-valued. We set

$$
C_{(0,}^{1}(\bar{\Omega})=\left\{u \in C^{1}(\bar{\Omega}) ; u=0 \text { in a neighborhood of } \overline{\partial_{1} \Omega}\right\} \text {. }
$$

The completion of $C_{(0)}^{1}(\bar{\Omega})$ with respect to the norm \|\|$_{1, p}$ is denoted by $V(\Omega)$. The space $V(\Omega)$ is reflexive and separable. The norm in $V(\Omega)$ is denoted by \|\|$_{v}$. Let $V^{\prime}(\Omega)$ be the dual space of $V(\Omega)$. As is well-known, Poincaré's inequality is valid for all functions in $V(\Omega)$, that is,

$$
\|u\|_{p} \leqq C\|\nabla u\|_{p}, \quad u \in V(\Omega) .
$$

Hereafter let $\alpha$ be a real number such that

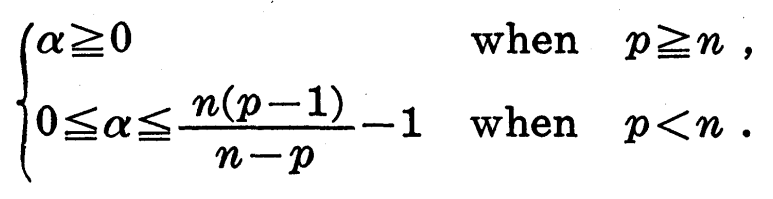

We denote by (, ) the inner product in $L^{2}(\Omega)$. For $u \in V(\Omega)$ we define 
$A(u)$ by the equality

$$
(A(u), v)=\left(|\nabla u|^{p-2} \nabla u, \nabla v\right)+\left(|u|^{\alpha} u, v\right), \quad v \in V(\Omega) .
$$

If $1<q<\infty$, let $q^{*}$ be the dual number of $q$, i.e., $q^{*}=q /(q-1)$. By Sobolev's imbedding theorem and $(0.2)$ the imbedding $V(\Omega) \rightarrow L^{(1+\alpha) p^{*}}(\Omega)$ is continuous. Hence we see that $A(u) \in V^{\prime}(\Omega)$.

We consider the equation $A(u)=f$ in $\Omega$, that is,

$$
\left(|\nabla u|^{p-2} \nabla u, \nabla v\right)+\left(|u|^{\alpha} u, v\right)=(f, v), \quad v \in V(\Omega) .
$$

The following inequalities hold: For $\xi, \eta \in R^{n}$

$$
\left(|\xi|^{p-2} \xi-|\eta|^{p-2} \eta, \xi-\eta\right) \geqq c_{0}|\xi-\eta|^{p}, \quad c_{0}>0,
$$

and for $a, b \in R^{1}$

$$
\left(|a|^{\alpha} a-|b|^{\alpha} b, a-b\right) \geqq 0 .
$$

It is known that with the aid of $(0.1),(0.4)$ and (0.5) the following proposition is obtained by the "monotonicity" method (cf., e.g., Chap. 2 in [11]).

Proposition 0.1. For $f \in V^{\prime}(\Omega)$ there is a unique solution $u \in V(\Omega)$ of (0.3).

For $x \in \boldsymbol{R}^{n}$ we denote by $\phi(x)$ the distance between $x$ and $S$. And we denote simply by $\partial^{k}$ any $k$-th order derivative with respect to the variables $x_{j}, 1 \leqq j \leqq n$. Our aim is to prove the following theorems.

THEOREM 1. Let $f$ be in $V^{\prime}(\Omega)$, and let $u \in V(\Omega)$ be a solution of (0.3). Then there is a positive number $\beta_{0}$ such that if $\phi^{1-\beta+\beta / p} f \in L^{p^{*}}(\Omega)$, where $0 \leqq \beta \leqq \beta_{0}$, it holds that $\phi^{-1-\beta / p} u, \phi^{-\beta / p} \partial u \in L^{p}(\Omega)$ and

$$
\left\|\phi^{-1-\beta / p} u\right\|_{p}+\left\|\phi^{-\beta / p} \partial u\right\|_{p} \leqq C\left(\left\|\phi^{1-\beta+\beta / p} f\right\|_{p^{*}}\right)^{1 /(p-1)} .
$$

Here $\beta_{0}$ and $C$ are independent of $f$.

REMARK. If $\phi^{1-\beta+\beta / p} f \in L^{p^{*}}(\Omega), \beta>0$, then $f \in V^{\prime}(\Omega)$ from Lemma 1.4 in the following section. Hence the assumption of $f \in V^{\prime}(\Omega)$ is superfluous in Theorem 1.

Let $\theta \cdot \nabla$ be a $C^{1}$ vector field on $\bar{\Omega}$ which is tangent to $\partial \Omega$ : if $\theta=$ $\left(\theta_{1}, \cdots, \theta_{n}\right), \theta \cdot \nabla=\sum_{i=1}^{n} \theta_{i} \partial_{x_{i}}$. Let $C^{\omega}$ be the class of analytic functions.

THEOREM 2. Suppose that $\partial \Omega$ and $S$ are both of class $C^{\infty}$. Let $f$ be 
in $W^{1, p^{*}}(\Omega)$, and let $u \in V(\Omega)$ be a solution of $(0.3)$. Then $(\theta \cdot \nabla)|\nabla u|^{p / 2} \in L^{1+\delta}(\Omega)$ for some $\delta>0$ and

$$
\left\|(\theta \cdot \nabla)|\nabla u|^{p / 2}\right\|_{1+\delta} \leqq C\left[\left(\|f\|_{1, p^{*}}\right)^{p^{*}}+\left(\|f\|_{p^{*}}\right)^{(2+\alpha) /(p-1)}\right]^{1 / 2},
$$

where $\delta$ and $C$ are independent of $f$.

THEOREM 3. Under the assumptions in Theorem 2, it holds that $\phi^{1-\delta}|\nabla u|^{p-1} \in W^{1, p^{*}}(\Omega)$ for some $\delta>0$ and

$$
\left\|\dot{\phi}^{1-\delta}|\nabla u|^{p-1}\right\|_{1, p^{*}} \leqq C\left[\|f\|_{1, p^{*}}+\left(\|f\|_{p^{*}}\right)^{(1+\alpha) /(p-1)}+\left(\|f\|_{p^{*}}\right)^{(2+\alpha) / p}\right],
$$

where $\delta$ and $C$ are independent of $f$.

Let $u \in V(\Omega)$ be a solution of (0.3). Then naturally $u=0$ on $\partial_{1} \Omega$. By Green's formula it follows that $|\nabla u|^{p-2} \sum_{j} \cos \left(\nu, x_{j}\right) \partial_{x_{j}} u=0$ on $\partial_{2} \Omega$ in the weak sense, where $\nu$ is the exterior normal of $\partial \Omega$ with respect to $\Omega$. Hence $\sum_{j} \cos \left(\nu, x_{j}\right) \partial_{x_{j}} u=0$ on $\partial_{2} \Omega$ in the same sense, so that $u$ satisfies the mixed boundary conditions of Dirichlet-Neumann type on $\partial \Omega$. Such a mixed boundary condition appears in the book of J. L. Lions [11] (cf. p. 345), where the existence of weak solutions for the non-stationary case was shown. Thus it seems meaningful for us to derive a regularity property up to the boundary for solutions of (0.3). Under mixed boundary conditions, nonlinear equations of another type or more general type were considered by several authors (cf., e.g., [1], [3], [5], [7], [12], [16], [18]). Here we do not state explicitly their results.

In 1968, E. Shamir [17] proved the regularity up to the boundary for solutions of linear elliptic equations, under general mixed boundary conditions. In this connection we note also the work in $\mathrm{H}$. Beirão da Veiga [20]. Recently, results analogous to [17] for linear parabolic equations have been proved by G. M. Lieberman [9]. The method in [17] is to construct a Green's function. Since it is not almost applicable to nonlinear equations, we have to use another method for (0.3). As an application of [17], M. K. V. Murthy and G. Stampacchia [13] proved the regularity for solutions of a single variational inequality with mixed boundary conditions. One of the authors and H. Nagase [4] have considered a system of variational inequalities and they have obtained an analogous result to one of the theorems in [13]. The method in [4] is, in a word, to use a parallel translation with a weight when one constructs a difference of differentiation for weak solutions.

The interior regularity for weak solutions of $A(u)=f$ was discussed by several authors (cf., e.g., [2], [8], [19]) and then the best result has been obtained. Next we consider the equation $A(u)=f$ under the Dirichlet 
boundary condition throughout $\partial \Omega$. In this case it is enough to replace $V(\Omega)$ with $W_{0}^{1, p}(\Omega)$ in (0.3). For this M. I. Vishik [21] first obtained a global regularity property by using Galerkin's method. In a series of papers G. N. Jakolev extended and improved the results in [21] (cf., e.g., [6]). His result is as follows: $|\nabla u|^{p-2} \partial u \in W^{1, p^{*}}(\Omega)$, if $f \in W^{1, p^{*}}(\Omega)$. In [6] the method of a simple parallel translation was used, which was already applied to linear elliptic equations by L. Nirenberg [14].

The argument in this paper is based on [14], [11, Chap. 2], [6] and particularly [4]. Theorem 2 is proved with the aid of Theorem 1 . We obtain Theorem 3 by proving the regularity of weak solutions along the normal direction of $\partial \Omega$.

\section{§1. Lemmas.}

Throughout this paper, the notation " $\rightarrow$ " means the weak convergence.

LEMMA 1.1 (Lions $\left[11\right.$, p. 12]). Let $u \in L^{q}(\Omega)(1<q<\infty)$ and suppose that $\left\{\left\|u_{j}\right\|_{q}\right\}$ is uniformly bounded and $u_{j} \rightarrow u$ pointwise a.e. in $\Omega$. Then $u_{j} \rightarrow u$ in $L^{q}(\Omega)$.

It is known that any $u \in W^{1, p}(\Omega)$ has its trace on $\partial \Omega$, so on $\partial_{1} \Omega$.

LEMMA 1.2. Let $u \in W^{1, p}(\Omega)$ and $u=0$ on $\partial_{1} \Omega$. Then $u \in V(\Omega)$.

Proof. For $R>0$ we set $\Sigma=\left\{x \in R^{n} ;|x|<R, x_{n}>0\right\}$. And we define

$$
\begin{aligned}
C^{1}{ }_{(0)}(\bar{\Sigma})=\left\{u \in C^{1}(\bar{\Sigma})\right. & ; u=0 \text { in a neighborhood } \\
& \text { of } \left.\{|x|=R\} \cup\left\{x_{n}=0, x_{n-1} \geqq 0\right\}\right\} .
\end{aligned}
$$

By a suitable partition of unity it is enough to prove the following assertion:

Let $u \in W^{1, p}(\Sigma)$. Let $u=0$ on $\left\{x_{n}=0, x_{n-1} \geqq 0\right\}$ and near $\{|x|=R\}$. Then there is a sequence $\left\{u_{j}\right\} \subset C_{(0)}^{1}(\bar{\Sigma})$ such that $u_{j} \rightarrow u$ in $W^{1, p}(\Sigma)$.

Let $u$ satisfy the assumptions in this proposition. We write $x^{\prime \prime}=$ $\left(x_{1}, \cdots, x_{n-2}\right)$. Since $u\left(x^{\prime \prime}, x_{n-1}+\varepsilon, x_{n}\right) \rightarrow u(x)$ in $W^{1, p}(\Sigma)$ as $\varepsilon \rightarrow+0$, we may assume that $u=0$ on $\left\{x_{n}=0, x_{n-1}>-\delta\right\}$ for some $\delta>0$.

Let us take a function $g\left(x_{n-1}\right)$ such that $g\left(x_{n-1}\right)=0$ for $x_{n-1} \leqq-\delta$ and $g\left(x_{n-1}\right)=-\left(x_{n-1}+\delta\right)^{2}$ for $x_{n-1}>-\delta$. And we set

$$
\tilde{\Sigma}=\{|x|<R\} \cap\left\{x_{n}>g\left(x_{n-1}\right)\right\}
$$

(see Figure 1). We extend $u$ throughout $\tilde{\Sigma}$ in such a way that $u=0$ in $\widetilde{\Sigma}-\Sigma$. Then $u \in W^{1, p}(\widetilde{\Sigma})$ obviously. Further we define an extension of $u$ in $\{|x|<R\}$ in such a way that $u=0$ on $\{|x|=R\}$ and $u$ is in $W^{1, p}(\{|x|<R\})$. 


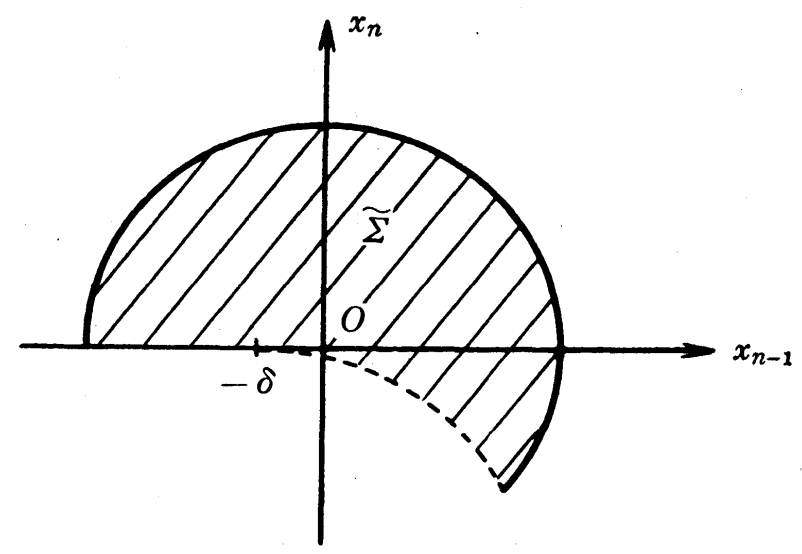

FIGURE 1

Then $u\left(x^{\prime \prime}, x_{n-1}, x_{n}-\varepsilon\right) \rightarrow u(x)$ in $W^{1, p}(\{|x|<R\})$ as $\varepsilon \rightarrow+0$. By using the mollifier, we can take a sequence $\left\{u_{j}\right\} \subset C_{t_{0}(}^{1}(\bar{\Sigma})$ satisfying $u_{j} \rightarrow u$ in $W^{1, p}(\Sigma)$. Thus the above assertion has been proved, so that we have completed the proof of our lemma.

Q.E.D.

Let $\boldsymbol{R}_{+}^{n}=\left\{\left(x_{1}, \cdots, x_{n}\right) ; x_{n}>0\right\}$, and let us define

$$
\begin{aligned}
C_{(0)}^{1}\left(\overline{\boldsymbol{R}_{+}^{n}}\right)=\left\{u \in C^{1}\left(\overline{\boldsymbol{R}_{+}^{n}}\right) ; u=0\right. \text { in a neighborhood of } \\
\\
\left.\quad\left\{x_{n}=0, x_{n-1} \geqq 0\right\} \text { and for sufficiently large }|x|\right\} .
\end{aligned}
$$

Hereafter we write $r=|x|$ and $\rho(x)=\left(x_{n-1}^{2}+x_{n}^{2}\right)^{1 / 2}$.

LEMMA 1.3. Let $t \neq 2$ and $q>1$. Then for any $u \in C_{(0)}^{1}\left(\overline{\boldsymbol{R}_{+}^{n}}\right)$ it holds that

$$
\int_{x_{n} \geq 0} \rho^{-t}|u|^{q} d x \leqq C \int_{x_{n} \geq 0} \rho^{q-t}|\nabla u|^{q} d x
$$

where $C$ is independent of $u$.

Proof. Obviously it is sufficient to prove only the case of $n=2$. Writing ' $=d / d r$, we have

$$
\begin{aligned}
\int_{0}^{\infty} r^{1-t}|u|^{q} d r & =\frac{-1}{2-t} \int_{0}^{\infty}\left(r^{2-t}\right)^{\prime}|u|^{q} d r \\
& =\frac{-q}{2-t} \int_{0}^{\infty} r^{2-t}|u|^{q-2} u u^{\prime} d r .
\end{aligned}
$$

Hence

$$
\int_{x_{2} \geq 0} \boldsymbol{r}^{-t}|u|^{q} d x \leqq C \int_{x_{2} \geq 0} \boldsymbol{r}^{1-t}|u|^{q-1}|\nabla u| d x
$$


(by Hölder's inequality)

$$
\leqq C\left(\int_{x_{2} \geq 0} r^{-t}|u|^{q} d x\right)^{(q-1) / q}\left(\int_{x_{2} \geq 0} r^{q-t}|\nabla u|^{q} d x\right)^{1 / q} .
$$

Applying Young's inequality, we complete the proof.

Q.E.D.

LEmma 1.4. Let $t \neq 2$ and $q>1$. Then for any $u \in C_{(0)}^{1}(\bar{\Omega})$ it holds that

$$
\int_{0} \phi^{-t}|u|^{q} d x \leqq C\left(\int_{\Omega} \phi^{q-t}|\nabla u|^{q} d x+\int_{\Omega} \phi^{q-t}|u|^{q} d x\right),
$$

where $C$ is independent of $u$.

Proof. Let $P_{0}$ be any fixed point in $S$. There are a neighborhood $U$ of $P_{0}$ and a $C^{1}$-homeomorphic mapping $\Phi$ from $U$ into $\left(y_{1}, \cdots, y_{n}\right)$-space such that

$$
\begin{array}{ll}
\Phi\left(P_{0}\right)=O, & \Phi(U \cap \Omega) \subset\left\{y_{n}>0\right\}, \\
\Phi(U \cap \partial \Omega) \subset\left\{y_{n}=0\right\}, & \Phi(U \cap S) \subset\left\{y_{n-1}=y_{n}=0\right\},
\end{array}
$$

and

$$
c \rho(y) \leqq \phi(x) \leqq c^{-1} \rho(y), \quad x \in U,
$$

where $c$ is some positive constant. Let $\eta \in C_{0}^{\infty}(U)$. Then from Lemma 1.3

$$
\int_{y_{n} \geq 0} \rho^{-t}|\eta u|^{q} d y \leqq C \int_{y_{n} \geq 0} \rho^{q-t}\left|\nabla_{y}(\eta u)\right|^{q} d y .
$$

Hence we have

$$
\begin{aligned}
\int_{\Omega} \phi^{-t}|\eta u|^{q} d x & \leqq C \int_{\Omega} \phi^{q-t}\left|\nabla_{x}(\eta u)\right|^{q} d x \\
& \leqq C\left(\int_{\Omega} \phi^{q-t}|\nabla u|^{q} d x+\int_{\Omega} \phi^{q-t}|u|^{q} d x\right) .
\end{aligned}
$$

By this inequality and a suitable partition of unity Lemma 1.4 is proved.

Q.E.D.

\section{§2. Propositions.}

For a subdomain $D$ of $\Omega$ we denote by \|\|$_{1, q, D}\left(\|\|_{q, D}\right)$ the norm in $W^{1, q}(D)\left(L^{q}(D)\right)$, respectively. We denote by $L_{10 c}^{q}(\Omega)$ the space of locally $q$-integrable functions in $\Omega$. And we define $W_{\mathrm{loc}}^{1, q}(\Omega)=\left\{u ; u, \partial u \in L_{\mathrm{loc}}^{q}(\Omega)\right\}$. 
The following proposition is due to M. I. Vishik [21], J. L. Lions [11, p. 112] and G. N. Jakolev [6]. We repeat its proof for the completeness. We follow mainly the method in [6]. However the inequality (2.3) is effective in our proof.

Proposition 2.1. Let $u$ be the solution in Proposition 0.1, and let $f$ be in $W_{10 \mathrm{oc}}^{1, p^{*}}(\Omega)$. Then $|\nabla u|^{p-2} \partial u \in W_{\mathrm{loc}}^{1, p^{*}}(\Omega)$. More precisely, for any subdomain $D$ with $\bar{D} \subset \Omega$ there is a constant $C$ depending on $D$ and $f$ such that

$$
\left\||\nabla u|^{p-2} \partial u\right\|_{1, p^{*}, D} \leqq C .
$$

PRoof. Let us fix any integer $i$ with $1 \leqq i \leqq n$. Let $h>0$ be sufficiently small. For any function $v$ we define

$$
\begin{aligned}
& v_{h}(x)=v\left(x_{1}, \cdots, x_{i-1}, x_{i}+h, x_{i+1}, \cdots, x_{n}\right), \\
& \left(D_{h} v\right)(x)=\frac{1}{h}\left(v_{h}(x)-v(x)\right) .
\end{aligned}
$$

Let us take a function $\zeta(x) \in C_{0}^{\infty}(\Omega)$. If $v \in W^{1, p}(\Omega)$, then $D_{h}\left(\zeta^{2} v\right) \in V(\Omega)$ obviously. Hence we have from (0.3)

$$
\left(|\nabla u|^{p-2} \nabla u, \nabla D_{h}\left(\zeta^{2} v\right)\right)+\left(|u|^{\alpha} u, D_{h}\left(\zeta^{2} v\right)\right)=\left(f, D_{h}\left(\zeta^{2} v\right)\right) .
$$

Evidently

$$
\begin{aligned}
& \left(|\nabla u|^{p-2} \nabla u, \nabla D_{h}\left(\zeta^{2} v\right)\right)=-\left(D_{-h}\left(|\nabla u|^{p-2} \nabla u\right), \nabla\left(\zeta^{2} v\right)\right), \\
& \left(|u|^{\alpha} u, D_{h}\left(\zeta^{2} v\right)\right)=-\left(D_{-h}\left(|u|^{\alpha} u\right), \zeta^{2} v\right)
\end{aligned}
$$

and

$$
\left(f, D_{h}\left(\zeta^{2} v\right)\right)=-\left(D_{-h} f, \zeta^{2} v\right) .
$$

Hence (2.1) becomes

$$
\begin{gathered}
\left(\zeta^{2} D_{-h}\left(|\nabla u|^{p-2} \nabla u\right), \nabla v\right)+2\left(\zeta D_{-h}\left(|\nabla u|^{p-2} \nabla u\right), v \nabla \zeta\right) \\
+\left(\zeta^{2} D_{-h}\left(|u|^{\alpha} u\right), v\right)=\left(\zeta^{2} D_{-h} f, v\right) .
\end{gathered}
$$

Replacing $v$ by $D_{-h} u$, we have

$$
\begin{gathered}
\left(\zeta^{2} D_{-h}\left(|\nabla u|^{p-2} \nabla u\right), D_{-h} \nabla u\right)+2\left(\zeta D_{-h}\left(|\nabla u|^{p-2} \nabla u\right), D_{-h} u \cdot \nabla \zeta\right) \\
+\left(\zeta^{2} D_{-h}\left(|u|^{\alpha} u\right), D_{-h} u\right)=\left(\zeta^{2} D_{-h} f, D_{-h} u\right) .
\end{gathered}
$$

By P. Lindqvist [10] the following inequality is valid: 


$$
\begin{aligned}
& \left(|\xi|^{p-2} \xi-|\eta|^{p-2} \eta, \xi-\eta\right) \\
& \geqq c_{0}\left(|\xi|^{p-2}+|\eta|^{p-2}\right)|\xi-\eta|^{2}, \quad \xi, \eta \in R^{n},
\end{aligned}
$$

where $c_{0}$ is a positive constant*). Hence we get

$$
\begin{aligned}
& \left(\zeta^{2} D_{-h}\left(|\nabla u|^{p-2} \nabla u\right), D_{-h} \nabla u\right) \\
& \geqq c_{0} \int_{\Omega}^{\zeta^{2}}\left(|\nabla u|^{p-2}+\left|\nabla u_{-h}\right|^{p-2}\right)\left|\nabla D_{-h} u\right|^{2} d x .
\end{aligned}
$$

If $\gamma \geqq 0$, it holds that for any $\xi, \eta \in \boldsymbol{R}^{n}$

$$
\left.|| \xi\right|^{r} \xi-|\eta|^{r} \eta|,||\xi|^{r+1}-|\eta|^{r+1}\left|\leqq C\left(|\xi|^{r}+|\eta|^{r}\right)\right| \xi-\eta \mid \text {, }
$$

which yields

$$
\left|D_{-h}\left(|\nabla u|^{p-2} \nabla u\right)\right| \leqq C\left(|\nabla u|^{p-2}+\left|\nabla u_{-h}\right|^{p-2}\right)\left|\nabla D_{-h} u\right| .
$$

Thus we have

$$
\begin{aligned}
& \left|\left(\zeta D_{-h}\left(|\nabla u|^{p-2} \nabla u\right), D_{-h} u \cdot \nabla \zeta\right)\right| \\
& \leqq C \int_{\Omega}|\zeta \nabla \zeta|\left(|\nabla u|^{p-2}+\left|\nabla u_{-h}\right|^{p-2}\right)\left|\nabla D_{-h} u\right|\left|D_{-h} u\right| d x \\
& \text { (by Hölder's inequality) }
\end{aligned}
$$

$$
\begin{aligned}
& \leqq C\left(\int_{\Omega} \zeta^{2}\left(|\nabla u|^{p-2}+\left|\nabla u_{-h}\right|^{p-2}\right)\left|\nabla D_{-h} u\right|^{2} d x\right)^{1 / 2} \\
& \quad \cdot\left(\int_{\Omega}|\nabla \zeta|\left(|\nabla u|^{p}+\left|\nabla u_{-h}\right|^{p}\right) d x\right)^{(p-2) / 2 p}\left(\int_{Q}|\nabla \zeta|\left|D_{-h} u\right|^{p} d x\right)^{1 / p}
\end{aligned}
$$

(by Young's inequality)

$$
\begin{aligned}
\leqq & \frac{c_{0}}{4} \int_{0} \zeta^{2}\left(|\nabla u|^{p-2}+\left|\nabla u_{-h}\right|^{p-2}\right)\left|\nabla D_{-h} u\right|^{2} d x \\
& +C\left[\int_{\Omega}|\nabla \zeta|\left(|\nabla u|^{p}+\left|\nabla u_{-h}\right|^{p}\right) d x+\int_{\Omega}|\nabla \zeta|\left|D_{-h} u\right|^{p} d x\right] .
\end{aligned}
$$

Now putting $v=u$ in $(0.3)$, we see that

$$
\left(\|\nabla u\|_{p}\right)^{p} \leqq(f, u) \leqq C\|f\|_{V^{\prime}}\|u\|_{V},
$$

so that

$$
\|\nabla u\|_{p} \leqq C\left(\|f\|_{V^{\prime}}\right)^{1 /(p-1)} .
$$

On the other hand it is clear that

*) Professor $H$. Nagase has informed us of this inequality. We are grateful to him for his kindness. 


$$
\int_{\Omega}|\nabla \zeta|\left|\nabla u_{-h}\right|^{p} d x, \int_{\Omega}|\nabla \zeta|\left|D_{-h} u\right|^{p} d x \leqq C \int_{\Omega}|\nabla u|^{p} d x
$$

From the above inequalities we obtain

$$
\begin{aligned}
& \left|\left(\zeta D_{-h}\left(|\nabla u|^{p-2} \nabla u\right), D_{-h} u \cdot \nabla \zeta\right)\right| \\
& \leqq \frac{c_{0}}{4} \int_{0} \zeta^{2}\left(|\nabla u|^{p-2}+\left|\nabla u_{-h}\right|^{p-2}\right)\left|\nabla D_{-h} u\right|^{2} d x+C\left(\|f\|_{V^{\prime}}\right)^{p^{*}},
\end{aligned}
$$

where $C$ is a constant depending on $\zeta$ and not on $h, f$. Since $\left\|\zeta D_{-h} u\right\|_{p} \leqq$ $C\|\nabla u\|_{p}$, it follows that

$$
\begin{aligned}
& \left|\left(\zeta^{2} D_{-h} f, D_{-h} u\right)\right| \leqq C\left\|\zeta D_{-h} f\right\|_{p^{*}}\|\nabla u\|_{p} \\
& (\text { from }(2.6)) \leqq C\left\|\zeta D_{-h} f\right\|_{p^{*}}\left(\|f\|_{V^{\prime}}\right)^{1 /(p-1)} .
\end{aligned}
$$

And we have from (0.5)

$$
\left(\zeta^{2} D_{-h}\left(|u|^{\alpha} u\right), D_{-h} u\right) \geqq 0 \text {. }
$$

Combining the above inequalities with (2.2) and (2.4) we conclude that

$$
\begin{aligned}
& \int_{\Omega} \zeta^{2}\left(|\nabla u|^{p-2}+\left|\nabla u_{-h}\right|^{p-2}\right)\left|\nabla D_{-h} u\right|^{2} d x \\
& \leqq C\left(\|f\|_{V^{\prime}}\right)^{1 /(p-1)}\left(\|f\|_{V^{\prime}}+\left\|\zeta D_{-h} f\right\|_{p^{*}}\right) .
\end{aligned}
$$

Here $\left\|\zeta D_{-k} f\right\|_{p^{*}} \leqq C$ from the assumption of $f \in W_{1 \text { oc }}^{1, p^{*}}(\Omega)$. On the other hand

$$
\begin{aligned}
& \left|D_{-h}\left(|\nabla u|^{p-2} \nabla u\right)\right|^{p^{*}} \\
& \leqq C\left(|\nabla u|^{p^{*}(p-2)}\left|\nabla D_{-h} u\right|^{p^{*}}+\left|\nabla u_{-h}\right|^{p^{*}(p-2)}\left|\nabla D_{-h} u\right|^{p^{*}}\right) .
\end{aligned}
$$

Using Hölder's inequality and noting that $p^{*}(p-2)=p\left(2-p^{*}\right)$, we have

$$
\begin{aligned}
& \int_{\Omega} \zeta^{p^{*}}\left|D_{-h}\left(|\nabla u|^{p-2} \nabla u\right)\right|^{p^{*}} d x \\
& \leqq C\left[\left(\int_{\Omega}|\nabla u|^{p} d x\right)^{\left(2-p^{*}\right) / 2}\left(\int_{\Omega} \zeta^{2}|\nabla u|^{p-2}\left|\nabla D_{-h} u\right|^{2} d x\right)^{p^{* / 2}}\right. \\
& \left.\quad+\left(\int_{\Omega}\left|\nabla u u_{-h}\right|^{p} d x\right)^{\left(2-p^{*} / 2\right.}\left(\int_{\Omega} \zeta^{2}\left|\nabla u_{-h}\right|^{p-2}\left|\nabla D_{-h} u\right|^{2} d x\right)^{p^{*} / 2}\right] .
\end{aligned}
$$

Combining (2.6), (2.7) with this inequality, we obtain

$$
\int_{\Omega} \zeta^{p^{*}}\left|D_{-h}\left(|\nabla u|^{p-2} \nabla u\right)\right|^{p^{*}} d x \leqq C,
$$

where $C$ depends on $f, \zeta$ and not on $h$. 
Let $D$ be any subdomain with $\bar{D} \subset \Omega$. Then from (2.8)

$$
\int_{D}\left|D_{-h}\left(|\nabla u|^{p-2} \nabla u\right)\right|^{p *} d x \leqq C,
$$

where $C$ depends on $f, D$ and not on $h$. Therefore for any $k$ with $1 \leqq k \leqq n$ there are a function $v_{k} \in L^{p^{*}}(D)$ and a positive sequence $\left\{h_{\nu}\right\}$ with $h_{\nu} \rightarrow 0(\nu \rightarrow \infty)$ such that

$$
D_{-k_{y}}\left(|\nabla u|^{p-2} \partial_{s_{k}} u\right) \rightarrow v_{k} \quad \text { in } L^{p^{*}}(D) \text {. }
$$

For any $\varphi \in C_{0}^{\infty}(D)$ we see that

$$
\begin{gathered}
\left(D_{-n_{\nu}}\left(|\nabla u|^{p-2} \partial_{\boldsymbol{x}_{k}} u\right), \phi\right)=-\left(|\nabla u|^{p-2} \partial_{\boldsymbol{x}_{k}} u, D_{\boldsymbol{h}_{\nu}} \varphi\right) \\
\rightarrow-\left(|\nabla u|^{p-2} \partial_{\boldsymbol{x}_{k}} u, \partial_{\boldsymbol{x}_{i}} \varphi\right),
\end{gathered}
$$

which implies that $v_{k}=\partial_{x_{i}}\left(|\nabla u|^{p-2} \partial_{x_{k}} u\right)$. Accordingly

$$
\left\|\partial_{x_{i}}\left(|\nabla u|^{p-2} \partial_{x_{k}} u\right)\right\|_{p^{*}, D} \leqq \varliminf_{\nu \rightarrow \infty}\left\|D_{-h_{\nu}}\left(|\nabla u|^{p-2} \partial_{x_{k}} u\right)\right\|_{p^{*}, D} \text {. }
$$

Combining this inequality with (2.9), we have finished the proof. Q.E.D.

REMARK. If we use another inequality in (2.5), the assertion in Proposition 2.1 holds also for $|\nabla u|^{p-1}$, that is, $|\nabla u|^{p-1} \in W_{10 c}^{1, p *}(\Omega)$. Hence by Sobolev's imbedding theorem we have

$$
|\nabla u|^{p-1} \in L_{100}^{n p^{*} /\left(n-p^{*}\right)}(\Omega) \text {. }
$$

Proposition 2.2. Under the assumptions in Proposition 2.1, $\left\{|\nabla u|^{p-2} D_{h} \partial u\right\}$ has a convergent subsequence in $L_{10 \mathrm{co}}^{p^{*}}(\Omega)$.

Proof. Let $q>1$, and let $q$ be so close to 1 if necessary. Let $D$ be a subdomain with $\bar{D} \subset \Omega$. Then by Hölder's inequality and (2.5),

$$
\begin{aligned}
& \left\|D_{\boldsymbol{h}}\left(|\nabla u|^{p-1} \partial u\right)\right\|_{q, D} \\
& \leqq C\left(\left(\left\|\nabla u_{h}\right\|_{p q /(2-q), D}\right)^{p / 2}+\left(\|\nabla u\|_{p q /(2-q), D}\right)^{p / 2}\right)\left\||\nabla u|^{(p-2) / 2} D_{h} \nabla u\right\|_{2, D} .
\end{aligned}
$$

Noting that $n(p-1) p^{*} /\left(n-p^{*}\right)>p$ and $p q /(2-q) \rightarrow p+0$ as $q \rightarrow 1+0$, we see that the right-hand side is uniformly bounded by $(2.10)$ and the proof of Proposition 2.1. Hence $|\nabla u|^{p-1} \partial u \in W_{10 c}^{1, q}(\Omega)$.

By the remark previous to this proposition, $\left\{D_{h}|\nabla u|^{p-1}\right\}$ is a convergent sequence in $L_{10 c}^{p *}(\Omega)$. Using (2.10) and Hölder's inequality, we see that $\left\{D_{h}|\nabla u|^{p-1} \cdot \partial u_{h}\right\}$ is a convergent sequence in $L_{100}^{q}(\Omega)$. Since

$$
|\nabla u|^{p-1} D_{h} \partial u=D_{h}\left(|\nabla u|^{p-1} \partial u\right)-D_{h}|\nabla u|^{p-1} \cdot \partial u_{h},
$$

$\left\{|\nabla u|^{p-1} D_{h} \partial u\right\}$ is also convergent in $L_{\mathrm{loc}}^{q}(\Omega)$. Thus there is a function $v \in L_{\mathrm{loc}}^{q}(\Omega)$ such that 


$$
|\nabla u|^{p-1} D_{h_{\nu}} \partial u \rightarrow v \quad \text { a.e. in } \Omega .
$$

From now on we denote by the same $\left\{h_{\nu}\right\}$ any subsequence of positive numbers tending to 0 . Setting $\widetilde{v}(x)=0$ if $|\nabla u(x)|=0$ and $\widetilde{v}(x)=v(x) /|\nabla u(x)|^{p / 2}$ if $|\nabla u(x)| \neq 0$, we obtain

$$
|\nabla u|^{(p-2) / 2} D_{h_{\nu}} \partial u \rightarrow \widetilde{v} \quad \text { a.e. in } \Omega .
$$

On the other hand from the proof of Proposition 2.1 there is a function $w \in L_{1 \mathrm{loc}}^{2}(\Omega)$ such that

$$
|\nabla u|^{(p-2) / 2} D_{h_{\nu}} \partial u \rightarrow w \quad \text { in } L_{1 \mathrm{oc}}^{2}(\Omega) .
$$

By applying Banach-Sacks theorem to the sequence $\left\{|\nabla u|^{(p-2) / 2} D_{h_{\nu}} \partial u\right\}$, we see that $w=\widetilde{v}$ in $\Omega$. If we repeat the argument in the book of Lions $[11$, p. 144], it follows that for any $\varepsilon>0$

$$
|\nabla u|^{(p-2) / 2} D_{h_{\nu}} \partial u \rightarrow w \quad \text { in } L_{10 \mathrm{co}}^{2-s}(\Omega),
$$

which completes the proof.

Q.E.D.

\section{§3. Proof of Theorem 1.}

Let $\left\{\delta_{j}\right\}$ be a monotone sequence of positive numbers decreasing to zero, and let us set $\omega_{j}=\left\{x ; \phi(x)<\delta_{j}\right\}$. Then $\left\{\omega_{j}\right\}$ is a sequence of neighborhoods of $S$ tending to $S$. Setting $\Omega_{j}=\Omega-\omega_{j}$, we define

$$
C_{(0)}^{1}\left(\bar{\Omega}_{j}\right)=\left\{u \in C^{1}\left(\bar{\Omega}_{j}\right) ; u=0 \text { near } \partial \omega_{j} \cup \overline{\partial_{1} \Omega}\right\} \text {. }
$$

Let $V\left(\Omega_{j}\right)$ be the completion of $C_{(0)}^{1}\left(\bar{\Omega}_{j}\right)$ with respect to the norm \|\|$_{1, p, \Omega_{j}}$. If we extend $u$ to be zero in $\omega_{j}$ for $u \in V\left(\Omega_{j}\right)$, then $u \in V(\Omega)$ and $u \in V\left(\Omega_{k}\right)$ for $k \geqq j$.

Proof of TheOREM 1. First we assume that $f \in W_{10 \mathrm{o}}^{1, p *}(\Omega)$. Replacing $\Omega$ with $\Omega_{j}$, by Proposition 0.1 we can find $u_{j} \in V\left(\Omega_{j}\right)$ such that $A\left(u_{j}\right)=f$ in $\Omega_{j}$, that is,

$$
\left(\left|\nabla u_{j}\right|^{p-2} \nabla u_{j}, \nabla v\right)+\left(\left|u_{j}\right|^{\alpha} u_{j}, v\right)=(f, v), \quad v \in V\left(\Omega_{j}\right) .
$$

Hereafter let $\beta$ be a positive number which is taken to be close to zero if necessary. Setting $v=\phi^{-\beta} u_{j}$ in (3.1) particularly, we have

$$
\begin{gathered}
\left(\phi^{-\beta}\left|\nabla u_{j}\right|^{p-2} \nabla u_{j}, \nabla u_{j}\right)+\left(u_{j}\left|\nabla u_{j}\right|^{p-2} \nabla \phi^{-\beta}, \nabla u_{j}\right) \\
+\left(\left|u_{j}\right|^{\alpha} u_{j}, \phi^{-\beta} u_{j}\right)=\left(f, \phi^{-\beta} u_{j}\right) .
\end{gathered}
$$

Since $|\nabla \phi|$ is bounded, it follows that 


$$
\begin{aligned}
& \left|\left(u_{j}\left|\nabla u_{j}\right|^{p-2} \nabla \phi^{-\beta}, \nabla u_{j}\right)\right| \\
& \leqq C \beta\left(\int_{\Omega} \phi^{-\beta}\left|\nabla u_{j}\right|^{p} d x\right)^{(p-1) / p}\left(\int_{\Omega} \phi^{-\beta-p}\left|u_{j}\right|^{p} d x\right)^{1 / p} \\
& \leqq C \beta\left(\int_{\Omega} \phi^{-\beta}\left|\nabla u_{j}\right|^{p} d x+\int_{\Omega} \phi^{-\beta-p}\left|u_{j}\right|^{p} d x\right)
\end{aligned}
$$

(by Lemma 1.4)

$$
\leqq C \beta\left(\int_{Q} \phi^{-\beta}\left|\nabla u_{j}\right|^{p} d x+\int_{0} \phi^{-\beta}\left|u_{j}\right|^{p} d x\right) \text {. }
$$

Using Poincaré's inequality and Lemma 1.4 again, we see that

$$
\left|\left(u_{j}\left|\nabla u_{j}\right|^{p-2} \nabla \phi^{-\beta}, \nabla u_{j}\right)\right| \leqq C \beta \int_{\Omega} \phi^{-\beta}\left|\nabla u_{j}\right|^{p} d x .
$$

Combining this with (3.2), we obtain

$$
\int_{Q} \phi^{-\beta}\left|\nabla u_{j}\right|^{p} d x \leqq C\left|\left(f, \phi^{-\beta} u_{j}\right)\right| \text {. }
$$

Clearly

$$
\left|\left(f, \phi^{-\beta} u_{j}\right)\right| \leqq\left\|\phi^{1-\beta+\beta / p} f\right\|_{p^{*}}\left\|\phi^{-1-\beta / p} u_{j}\right\|_{p} .
$$

From Poincaré's inequality and Lemma 1.4

$$
\begin{aligned}
\left\|\phi^{-1-\beta / p} u_{j}\right\|_{p} & \leqq C\left(\left\|\phi^{-\beta / p} \nabla u_{j}\right\|_{p}+\left\|\phi^{-\beta / p} u_{j}\right\|_{p}\right) \\
& \leqq C\left\|\phi^{-\beta / p} \nabla u_{j}\right\|_{p} .
\end{aligned}
$$

These two inequalities and (3.3) yield

$$
\left\|\phi^{-1-\beta / p} u_{j}\right\|_{p}+\left\|\phi^{-\beta / p} \nabla u_{j}\right\|_{p} \leqq C\left(\left\|\phi^{1-\beta+\beta / p} f\right\|_{\left.p^{*}\right)^{1 /(p-1)}} .\right.
$$

Hereafter let us denote by the same notation $\left\{u_{k}\right\}$ any subsequence of $\left\{u_{j}\right\}$. By (3.4) and Sobolev's compact imbedding theorem there is a function $u \in L^{p}(\Omega)$ such that

$$
u_{k} \rightarrow u \quad \text { in } L^{p}(\Omega) \text {. }
$$

Combining (3.4) with (3.5), we have

$$
\phi^{-1-\beta / p} u_{k} \rightarrow \phi^{-1-\beta / p} u \quad \text { in } L^{p}(\Omega)
$$

and for any $i$ with $1 \leqq i \leqq n$

$$
\phi^{-\beta / p} \partial_{w_{i}} u_{k} \rightarrow \phi^{-\beta / p} \partial_{x_{i}} u \quad \text { in } L^{p}(\Omega) \text {. }
$$

From this we see that $u \in V(\Omega)$. Further from (3.4) again we have 


$$
\left\|\phi^{-1-\beta / p} u\right\|_{p}+\left\|\phi^{-\beta / p} \nabla u\right\|_{p} \leqq C\left(\left\|\phi^{1-\beta+\beta / p} f\right\|_{p^{*}}\right)^{1 /(p-1)} \text {. }
$$

Let $D$ be any subdomain with $\bar{D} \subset \Omega$. Since we have assumed that $f \in W_{10 \mathrm{co}}^{1, p^{*}}(\Omega)$, we can apply Proposition 2.1. Thus there is a positive integer $j_{0}\left(=j_{0}(D)\right)$ such that

$$
\left\|\left|\nabla u_{j}\right|^{p-2} \nabla u_{j}\right\|_{1, p^{*}, D} \leqq C
$$

for $j \geqq j_{0}$, where $C$ depends on $f, D$ and not on $j$. Hence we can apply Sobolev's compact imbedding theorem to the sequence $\left\{\left|\nabla u_{j}\right|^{p-2} \nabla u_{j}\right\}$ as follows: for each $i, 1 \leqq i \leqq n$, there is a function $g_{i} \in L_{\mathrm{loc}}^{p *}(\Omega)$ such that for any $D$ with $\bar{D} \subset \Omega$

$$
\begin{cases}\left|\nabla u_{k}\right|^{p-2} \partial_{x_{i}} u_{k} \rightarrow g_{i} & \text { in } L^{p^{*}}(D) \\ \left|\nabla u_{k}\right|^{p-2} \partial_{x_{i}} u_{k} \rightarrow g_{i} & \text { a.e. in } \Omega .\end{cases}
$$

This implies that $g_{i}=\left(\sum_{l=1}^{n} h_{l}^{2}\right)^{(p-2) / 2} h_{i}$ for some functions $\left\{h_{i}\right\}$ and

$$
\partial_{x_{i}} u_{k} \rightarrow h_{i} \quad \text { a.e. in } \Omega \text {. }
$$

Clearly $h_{i} \in L^{p}(D)$, so that we have by Lemma 1.1

$$
\partial_{x_{i}} u_{k} \rightarrow h_{i} \quad \text { in } L^{p}(D) \text {. }
$$

From this and (3.5) we see that $h_{i}=\partial_{x_{i}} u$. Therefore it follows from (3.7) that

$$
\left|\nabla u_{k}\right|^{p-2} \partial_{x_{i}} u_{k} \rightarrow|\nabla u|^{p-2} \partial_{x_{i}} u \quad \text { a.e. in } \Omega
$$

and

$$
\left(\left|\nabla u_{k}\right|^{p-2} \partial_{x_{i}} u_{k}, \varphi\right) \rightarrow\left(|\nabla u|^{p-2} \partial_{x_{i}} u, \varphi\right), \quad \phi \in C_{0}^{\infty}(\Omega) \text {. }
$$

Since $\left\{\left|\nabla u_{j}\right|^{p-2} \partial_{x_{i}} u_{j}\right\}$ are uniformly bounded in $L^{p^{*}}(\Omega)$, it holds from (3.9) that

$$
\left|\nabla u_{k}\right|^{p-2} \partial_{x_{i}} u_{k} \rightarrow|\nabla u|^{p-2} \partial_{x_{i}} u \quad \text { in } L^{p^{*}}(\Omega) \text {. }
$$

Hence we obtain

$$
\left(\left|\nabla u_{k}\right|^{p-2} \nabla u_{k}, \nabla v\right) \rightarrow\left(|\nabla u|^{p-2} \nabla u, \nabla v\right), \quad v \in C_{(0)}^{1}(\Omega) .
$$

The following inequality is valid from Sobolev's imbedding theorem and the assumption (0.2):

$$
\left\|\left|u_{j}\right|^{\alpha} u_{j}\right\|_{p^{*}} \leqq C\left(\left\|u_{j}\right\|_{V}\right)^{1+\alpha} .
$$

From this and (3.4) we see that $\left\{\left\|\left|u_{j}\right|^{\alpha} u_{j}\right\|_{p^{*}}\right\}$ are uniformly bounded. More 


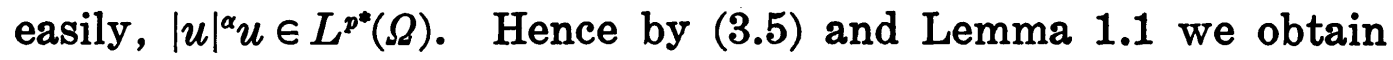

$$
\left(\left|u_{k}\right|^{\alpha} u_{k}, v\right) \rightarrow\left(|u|^{\alpha} u, v\right), \quad v \in C_{(0)}^{1}(\bar{\Omega}) .
$$

Let us fix any $v \in C_{(0)}^{1}(\bar{\Omega})$. Then from (3.1) there is a positive integer $j_{1}$ such that for $j \geqq j_{1}$

$$
\left(\left|\nabla u_{j}\right|^{p-2} \nabla u_{j}, \nabla v\right)+\left(\left|u_{j}\right|^{\alpha} u_{j}, v\right)=(f, v) .
$$

Combining (3.10), (3.11) with this equality we conclude that

$$
\left(|\nabla u|^{p-2} \nabla u, \nabla v\right)+\left(|u|^{\alpha} u, v\right)=(f, v), \quad v \in C_{(0)}^{1}(\bar{\Omega}),
$$

which holds also for any $v \in V(\Omega)$ as easily seen. That is, $u$ is the solution itself in Theorem 1 . In view of (3.6), Theorem 1 is correct under the assumption of $f \in W_{1 \mathrm{ic}}^{1, p^{*}}(\Omega)$.

Next let us remove the assumption of $f \in W_{1 \text { loc }}^{1, p^{*}}(\Omega)$. We take a sequence $\left\{g_{j}\right\} \subset C_{0}^{1}(\Omega)$ such that $g_{j} \rightarrow \phi^{1-\beta+\beta / p} f$ in $L^{p^{*}(\Omega)}$. If we set $f_{j}=$ $\phi^{\beta-1-\beta / p} g_{j}$, then $\phi^{1-\beta+\beta / p} f_{j} \rightarrow \phi^{1-\beta+\beta / p} f$ in $L^{p^{*}}(\Omega)$. It follows by Lemma 1.4 that for $v \in C_{(0)}^{1}(\bar{\Omega})$

$$
\begin{aligned}
\left\|\phi^{\beta-1-\beta / p} v\right\|_{p} & \leqq C\left(\left\|\phi^{\beta-\beta / p} \nabla v\right\|_{p}+\left\|\phi^{\beta-\beta / p} v\right\|_{p}\right) \\
& \leqq C\|v\|_{V},
\end{aligned}
$$

so that

$$
\left|\left(f_{j}-f, v\right)\right| \leqq C\left\|\phi^{1-\beta+\beta / p}\left(f_{j}-f\right)\right\|_{p^{*}}\|v\|_{v} .
$$

This implies that

$$
\left\|f_{j}-f\right\|_{V^{\prime}} \leqq C\left\|\phi^{1-\beta+\beta / p}\left(f_{j}-f\right)\right\|_{p^{*}} .
$$

Hence $f_{j} \rightarrow f$ in $V^{\prime}(\Omega)$. Let $u_{j} \in V(\Omega)$ be the solution of $A\left(u_{j}\right)=f_{j}$ in $\Omega$. Then Theorem 1 is valid for each $u_{j}$ from the first half, so that

$$
\left\|\phi^{-1-\beta / p} u_{j}\right\|_{p}+\left\|\phi^{-\beta / p} \nabla u_{j}\right\|_{p} \leqq C\left(\left\|\phi^{1-\beta+\beta / p} f_{j}\right\|_{p^{*}}\right)^{1 /(p-1)} .
$$

It is obvious that for any $v \in V(\Omega)$

$$
\left(\left|\nabla u_{j}\right|^{p-2} \nabla u_{j}-|\nabla u|^{p-2} \nabla u, \nabla v\right)+\left(\left|u_{j}\right|^{\alpha} u_{j}-|u|^{\alpha} u, v\right)=\left(f_{j}-f, v\right) \text {. }
$$

Setting $v=u_{j}-u$ in this equality, we have from (0.4) and (0.5)

$$
c_{0}\left(\left\|\nabla\left(u_{j}-u\right)\right\|_{p}\right)^{p} \leqq\left\|f_{j}-f\right\|_{V}\left\|u_{j}-u\right\|_{V},
$$

so that 


$$
\left.\left\|u_{j}-u\right\|_{V} \leqq C\left(\left\|f_{j}-f\right\|_{V}\right)^{1 /(p-1)}\right) .
$$

This yields that $u_{j} \rightarrow u$ in $V(\Omega)$. Therefore it follows from (3.12) that

$$
\phi^{-\beta / p} \partial_{x_{i}} u_{k} \rightarrow \phi^{-\beta / p} \partial_{x_{i}} u \text { in } L^{p}(\Omega), \quad i=1, \cdots, n .
$$

Accordingly,

$$
\left\|\phi^{-\beta / p} \nabla u\right\|_{p} \leqq C\left(\left\|\phi^{1-\beta+\beta / p} f\right\|_{p *}\right)^{1 /(p-1)} .
$$

We have similarly

$$
\left\|\phi^{-1-\beta / p} u\right\|_{p} \leqq C\left(\left\|\phi^{1-\beta+\beta / p} f\right\|_{p^{*}}\right)^{1 /(p-1)} .
$$

Thus we have finished the proof of Theorem 1 .

Q.E.D.

\section{§4. Parallel translations with a weight.}

The content of this section is due to [4]. The descriptions of Lemmas 4.2 and 4.3 are slightly different from those in [4].

For some time we consider our lemma in the upper half space $\left\{x_{n} \geqq 0\right\}$. We denote $\left\{x_{n}>0\right\}$ by $\boldsymbol{R}_{+}^{n}$. Let $\gamma$ be any fixed real number with $0<\gamma<1$, which may be chosen so close to 1 . Let us define $\tilde{\rho}(x)=\left(x_{n-1}^{2}+x_{n}^{27}\right)^{1 / 2}$. We write often $\tilde{\rho}(x)$ simply with $\tilde{\rho}$. It is easily seen that

$$
\left|\partial_{x_{n-1}} \tilde{\rho}\right| \leqq C, \quad\left|\partial_{x_{n}} \tilde{\rho}\right| \leqq C \tilde{\rho}^{1-1 / r} .
$$

We define the following mapping from $\boldsymbol{R}_{+}^{n}$ into itself:

$$
\Phi_{h}:\left\{\begin{array}{l}
y_{j}=x_{j} \quad \text { if } j \neq n-1 \\
y_{n-1}=x_{n-1}+h \tilde{\rho}
\end{array}\right.
$$

where $h$ is a sufficiently small positive number. Hereafter we suppose that the $y$-variable is always connected with the $x$-variable by the equality $y=\Phi_{h}(x)$. Thus $\tilde{\rho}(y)=\tilde{\rho}\left(\Phi_{h}(x)\right)$. There is a positive constant $c$ such that

$$
c \tilde{\rho}(x) \leqq \tilde{\rho}(y) \leqq c^{-1} \tilde{\rho}(x), \quad x \in \overline{R_{+}^{n}} .
$$

In fact the inequality on the right is trivial. That on the left is easily seen from

$$
\tilde{\rho}^{2} \leqq 2\left(y_{n-1}^{2}+y_{n}^{2 \gamma}+h^{2} \tilde{\rho}^{2}\right) .
$$

By an easy computation we have 


$$
\left(\begin{array}{c}
\partial_{x_{1}} y_{1} \cdots \cdots \partial_{x_{1}} y_{n} \\
\cdots \cdots \\
\partial_{x_{n}} y_{1} \cdots \partial_{x_{n}} y_{n}
\end{array}\right)=\left(\begin{array}{ccc}
1 & & 0 \\
\ddots & 1 & 0 \\
& 1+h \partial_{x_{n-1}} \tilde{\rho} & 0 \\
0 & h \partial_{x_{n}} \tilde{\rho} & 1
\end{array}\right),
$$

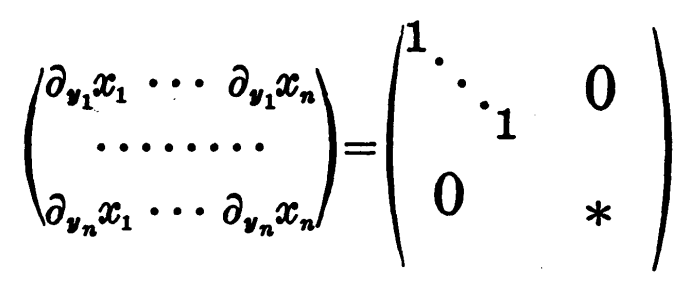

where

$$
*=\frac{1}{1+h \partial_{x_{n-1}} \tilde{\rho}}\left(\begin{array}{cc}
1 & 0 \\
-h \partial_{x_{n}} \tilde{\rho} & 1+h \partial_{z_{n-1}} \tilde{\rho}
\end{array}\right) \text {. }
$$

From (4.1) and (4.3) we get

$$
\left|\partial_{y_{n-1}} \tilde{\rho}\right| \leqq C, \quad\left|\partial_{y_{n}} \tilde{\rho}\right| \leqq C \tilde{\rho}^{1-1 / r}
$$

Let $J_{h}$ be the Jacobian of $\Phi_{h}$. We can write $J_{h}=1+h \partial_{x_{n-1}} \tilde{\rho}$, so that $c \leqq J_{h} \leqq c^{-1}$ for some positive constant $c$. It is easy to see that $\Phi_{h}$ is a one-to-one mapping from $\overline{R_{+}^{n}}$ onto itself.

Let us write $x^{\prime \prime}=\left(x_{1}, \cdots, x_{n-2}\right) \quad\left(y^{\prime \prime}=\left(y_{1}, \cdots, y_{n-2}\right)\right)$ for $x=\left(x_{1}, \cdots, x_{n}\right)$ $\left(y=\left(y_{1}, \cdots, y_{n}\right)\right)$, respectively. We define

$$
\begin{aligned}
& \left(S_{h} u\right)(x)=u\left(x^{\prime \prime}, x_{n-1}+h \tilde{\rho}, x_{n}\right), \\
& \left(T_{h} u\right)(y)=u\left(y^{\prime \prime}, y_{n-1}-h \tilde{\rho}, y_{n}\right) .
\end{aligned}
$$

Namely, $\left(S_{h} u\right)(x)=u(y)$ and $\left(T_{h} u\right)(y)=u(x)$. Further we define

$$
\begin{aligned}
& \left(P_{h} u\right)(x)=h^{-1}\left(\left(S_{h} u\right)(x)-u(x)\right), \\
& \left(Q_{h} u\right)(y)=h^{-1}\left(\left(T_{h} u\right)(y)-u(y)\right) .
\end{aligned}
$$

We have always $\left(Q_{h} u\right)(y)=-\left(P_{h} u\right)(x)$. From now on, $\left(S_{h} u\right)(x),\left(T_{h} u\right)(y)$, $\left(P_{h} u\right)(x)$ and $\left(Q_{h} u\right)(y)$ are often written simply by $S_{h} u, T_{h} u, P_{h} u$ and $Q_{h} u$, respectively. Clearly

$$
\begin{aligned}
& P_{h}(u v)=S_{h} u \cdot P_{h} v+v P_{h} u, \\
& Q_{h}(u v)=T_{h} u \cdot Q_{h} v+v Q_{h} u
\end{aligned}
$$

If we define

$$
F_{k}\left(\nabla_{z} u\right)=h^{-1}\left(\nabla_{z}\left(S_{h} u\right)-S_{h} \nabla_{z} u\right)
$$


and

$$
G_{h}\left(\nabla_{y} u\right)=h^{-1}\left(\nabla_{y}\left(T_{h} u\right)-T_{h} \nabla_{y} u\right),
$$

then it is easily seen that

$$
\left\{\begin{array}{l}
\left|\boldsymbol{F}_{h}\left(\nabla_{x} u\right)\right| \leqq C \tilde{\rho}^{1-1 / \gamma}\left|S_{h} \partial_{x_{n-1}} u\right| \\
\left|G_{h}\left(\nabla_{y} u\right)\right| \leqq C \tilde{\rho}^{1-1 / \gamma}\left|T_{h} \partial_{y_{n-1}} u\right|
\end{array}\right.
$$

on any bounded subset of $\overline{\boldsymbol{R}_{+}^{n}}$, where $C$ is independent of $h$ and $u$.

For the time being, let us denote by $($,$) and \|\|_{q}$ the inner product in $L^{2}\left(\boldsymbol{R}_{+}^{n}\right)$ and the norm in $L^{q}\left(\boldsymbol{R}_{+}^{n}\right)$, respectively. Let $(,)_{x}\left((,)_{y}\right)$ be the inner product in $L^{2}\left(\boldsymbol{R}_{+}^{n}\right)$ with respect to the $x$-variable $(y$-variable), respectively.

Lemma 4.1. For arbitrary functions $u, v$ it holds that

$$
\left(u, P_{h} v\right)_{x}=\left(Q_{h} u, v\right)_{y}+\left(K_{h} T_{h} u, v\right)_{y},
$$

where $K_{h}(y)=-\partial_{x_{n-1}} \tilde{\rho} /\left(1+h \partial_{x_{n-1}} \tilde{\rho}\right)$.

Proof. We see that

$$
\begin{aligned}
& \left(u, P_{h} v\right)_{x}=h^{-1}\left(\int_{R_{+}^{n}} u(x) v(y) d x-\int_{R_{+}^{n}} u(x) v(x) d x\right), \\
& \int_{R_{+}^{n}} u(x) v(x) d x=\int_{R_{+}^{n}} u(y) v(y) d y
\end{aligned}
$$

and

$$
\int_{R_{+}^{n}} u(x) v(y) d x=\int_{R_{+}^{n}} T_{h} u \cdot v J_{h}^{-1} d y .
$$

Obviously $J_{h}^{-1}=1+h K_{h}$, so that we obtain the required equality. Q.E.D.

LEMmA 4.2. Let $1 \leqq q<\infty$, and let $u \in W^{1, q}\left(\boldsymbol{R}_{+}^{n}\right)$. Then it holds that

$$
\left\|\tilde{\rho}^{-1} P_{h} u\right\|_{q} \leqq C\left\|\partial_{x_{n-1}} u\right\|_{q}
$$

and

$$
\left\|\tilde{\rho}^{-1} Q_{h} u\right\|_{q} \leqq C\left\|\partial_{y_{n-1}} u\right\|_{q},
$$

where $C$ is independent of $h$ and $u$.

Proof. Without loss of generality we may assume that $u \in C^{1}\left(\overline{R_{+}^{n}}\right)$ and $u=0$ for large $|x|$. Since 


$$
\left(P_{h} u\right)(x)=\tilde{\rho} \int_{0}^{1}\left(\partial_{x_{n-1}} u\right)\left(x^{\prime \prime}, x_{n-1}+t h \tilde{\rho}, x_{n}\right) d t,
$$

it follows that

$$
\begin{aligned}
\left(\left\|\tilde{\rho}^{-1} P_{h} u\right\|_{q}\right)^{q} & \leqq \int_{0}^{1} \int_{Q}\left|\left(\partial_{x_{n-1}} u\right)\left(x^{\prime \prime}, x_{n-1}+t h \tilde{\rho}, x_{n}\right)\right|^{q} d x d t \\
& =\int_{0}^{1} \int_{Q}\left|\left(\partial_{y_{n-1}} u\right)(y)\right|^{q} J_{t h}^{-1} d y d t \\
& \leqq C\left(\left\|\partial_{x_{n-1}} u\right\|_{q}\right)^{q},
\end{aligned}
$$

which proves the first inequality. The second inequality is reduced to the first one by using (4.2).

LEMMA 4.3. Let $1 \leqq q<\infty$. Let $u \in W^{1, q}\left(\boldsymbol{R}_{+}^{n}\right)$. Then

$$
\left\|\tilde{\rho}^{-1} P_{h} u-\partial_{x_{n-1}} u\right\|_{q} \rightarrow 0
$$

and

$$
\left\|\tilde{\rho}^{-1} Q_{h} u+\partial_{y_{n-1}} u\right\|_{q} \rightarrow 0 \quad \text { as } h \rightarrow 0 .
$$

Proof. By Lemma 4.2 it is enough to assume that $u \in C^{1}\left(\overline{\boldsymbol{R}_{+}^{n}}\right)$ and $u=0$ for large $|x|$. Similarly to the proof of Lemma 4.2 we have

$$
\begin{aligned}
& \left(\left\|\tilde{\rho}^{-1} P_{h} u-\partial_{x_{n-1}} u\right\|_{q}\right)^{q} \\
& \leqq \int_{0}^{1} \int_{\Omega}\left|\left(\partial_{x_{n-1}} u\right)\left(x^{\prime \prime}, x_{n-1}+t h \tilde{\rho}, x_{n}\right)-\left(\partial_{x_{n-1}} u\right)(x)\right|^{q} d x d t .
\end{aligned}
$$

Clearly the right-hand side tends to zero as $h \rightarrow 0$, so that the first inequality has been proved. The second inequality is reduced to the first one, because $\|(\nabla u)(y)-(\nabla u)(x)\|_{q} \rightarrow 0$ as $h \rightarrow 0$.

Q.E.D.

$\S 5$. A localization of $\bar{\Omega}$.

Let $\Omega$ be the domain in Theorem 2. In this section we prepare a localization of $\partial \Omega$ near $S$. We denote the origin simply by $O$ and we write $x^{\prime}=\left(x_{1}, \cdots, x_{n-1}\right)$. The origin in the $x^{\prime}$-space is denoted also by the same $O$.

LEMma 5.1. Let $P_{0}$ be any fixed point on $\partial \Omega$. Then there is a neighborhood $U$ of $P_{0}$ and a function $u \in C^{\omega}(U)$ such that

$$
u=0 \text { on } \partial \Omega \cap U, \quad u>0 \text { in } \Omega \cap U
$$

and

$$
|\nabla u|=1 \text { in } U \text {. }
$$


Proof. We may asssume that $P_{0}=O$. We consider our lemma in a neighborhood of $O$. By an orthogonal coordinate transformation it is sufficient to assume that $\Omega$ and $\partial \Omega$ are expressed by $x_{n}>\psi\left(x^{\prime}\right)$ and $x_{n}=$ $\psi\left(x^{\prime}\right)$, respectively, where $\psi(O)=0, \partial_{x_{i}} \psi(O)=0$ for $i, 1 \leqq i \leqq n-1$, and $\psi\left(x^{\prime}\right)$ is analytic near $O$.

We take the following coordinate transformation

Then

$$
y_{i}=x_{i} \quad(i \neq n), \quad y_{n}=x_{n}-\psi\left(x^{\prime}\right) .
$$

$$
\begin{aligned}
& \partial_{x_{i}} u=\partial_{y_{i}} u-\partial_{x_{i}} \psi \cdot \partial_{y_{n}} u \quad(i \neq n), \\
& \partial_{x_{n}} u=\partial_{y_{n}} u,
\end{aligned}
$$

so that the equation $\left|\nabla_{x} u\right|^{2}=1$ is equivalent to

$$
\left(1+\sum_{i=1}^{n-1}\left(\partial_{x_{i}} \psi\right)^{2}\right)\left(\partial_{y_{n}} u\right)^{2}+\sum_{i=1}^{n-1}\left(\partial_{y_{i}} u\right)^{2}-2\left(\sum_{i=1}^{n-1} \partial_{x_{i}} \psi \cdot \partial_{y_{i}} u\right) \partial_{y_{n}} u=1
$$

If $u>0$ for $y_{n}>0$, this equation becomes

$$
\begin{aligned}
\partial_{y_{n}} u= & \left(1+\sum_{i=1}^{n-1}\left(\partial_{x_{i}} \psi\right)^{2}\right)^{-1}\left[\sum_{i=1}^{n-1} \partial_{x_{i}} \psi \cdot \partial_{y_{i}} u\right. \\
& +\sqrt{\left.\left(\sum_{i=1}^{n-1} \partial_{x_{i}} \psi \cdot \partial_{y_{i}} u\right)^{2}+\left(1+\sum_{i=1}^{n-1}\left(\partial_{x_{i}} \psi\right)^{2}\right)\left(1-\sum_{i=1}^{n-1}\left(\partial_{y_{i}} u\right)^{2}\right)\right]} .
\end{aligned}
$$

The initial condition for $u$ is written by

$$
u\left(y^{\prime}, 0\right)=0 \text {. }
$$

We shall solve the Cauchy problem (5.1) with the initial condition (5.2). We refer to the book of I. G. Petrovskii [15]. Setting $P_{i}=\partial_{y_{i}} u$ for $i \neq n$, we write the right-hand side of (5.1) with $F\left(y^{\prime}, p_{1}, \cdots, p_{n-1}\right)$. By differentiating the both sides of (5.1), we have

$$
\partial_{y_{n}}^{2} u=\sum_{i=1}^{n-1} \partial_{p_{i}} F \cdot \partial_{y_{n}} \partial_{y_{i}} u \text {. }
$$

If we set $u_{0}=u$ and $u_{i}=\partial_{y_{i}} u$ for $i, 1 \leqq i \leqq n$, the Cauchy problems (5.1'), (5.2) become

$$
\left\{\begin{array}{l}
\partial_{y_{n}} u_{0}=u_{n} \\
\partial_{y_{n}} u_{1}=\partial_{y_{1}} u_{n} \\
\cdots \cdots \cdots \\
\partial_{y_{n}} u_{n-1}=\partial_{y_{n-1}} u_{n} \\
\partial_{y_{n}} u_{n}=\sum_{i=1}^{n-1}\left(\partial_{p_{i}} F\right)\left(y^{\prime}, u_{1}, \cdots, u_{n-1}\right) \partial_{y_{i}} u_{n},
\end{array}\right.
$$




$$
\left\{\begin{array}{l}
u_{0}\left(y^{\prime}, 0\right)=u_{1}\left(y^{\prime}, 0\right)=\cdots=u_{n-1}\left(y^{\prime}, 0\right)=0 \\
u_{n}\left(y^{\prime}, 0\right)=F\left(y^{\prime}, 0, \cdots, 0\right) .
\end{array}\right.
$$

If (5.1") and (5.2 ) are solvable, we easily see that $u=u_{0}$ is a solution of (5.1) and (5.2). By Cauchy-Kowalevski's theorem there is a solution $u$ of (5.1") and (5.2') such that $u$ is analytic near $O$ (cf., e.g., [15]), so that we complete the proof.

Q.E.D.

LEMma 5.2. Let $U$ be a neighborhood of $O$ in $\boldsymbol{R}^{n}$. Let $\left(v_{1}, \cdots, v_{n}\right) \epsilon$ $\left[C^{\omega}(U)\right]^{n}$, and let $\left(v_{1}, \cdots, v_{n}\right) \neq O$ in $U$. Then there are another neighborhood $U^{\prime}$ of $O$ and vector functions $\left(a_{i 1}, \cdots, a_{i n}\right) \in\left[C^{\omega}\left(U^{\prime}\right)\right]^{n}, i=1, \cdots, n-1$, such that $\left(a_{i 1}, \cdots a_{i n}\right) \neq O, \sum_{k=1}^{n} a_{i k} v_{k}=0$ in $U^{\prime}$ and $\sum_{k=1}^{n} a_{i k} a_{j k}=0$ in $U^{\prime}$ if $i \neq j$.

Proof. In the following we often omit the phrase "in a neighborhood of $O$ ". Let us prove our lemma by induction on $n$.

Let $n=2$. Since $\left(v_{1}, v_{2}\right) \neq O$, we may assume that $v_{1} \neq 0$. It is enough to take $a_{2}=1$ and $a_{1}=-a_{2} v_{2} / v_{1}$. Next let us assume that our lemma is correct. Then we shall prove it, when $n$ is replaced with $n+1$.

Without loss of generality we may suppose that $\left(v_{1}, \cdots, v_{n}\right) \neq 0$. From our assumption there are vector functions $\left(a_{i 1}, \cdots, a_{i n}\right)(\neq 0) \in\left[C^{\omega}\right]^{n}, i=$ $1, \cdots, n-1$, which are orthogonal to each other and so to $\left(v_{1}, \cdots, v_{n}\right)$. Thus $n$ vector functions $\left(v_{1}, \cdots, v_{n}\right)$ and $\left(a_{i 1}, \cdots, a_{i n}\right), i=1, \cdots, n-1$, are linearly independent, which implies that

$$
\left|\begin{array}{ccc}
a_{11} & \cdots & a_{1 n} \\
\cdots & \cdots & a^{\prime} \\
a_{n-1,1} & \cdots & a_{n-1, n} \\
v_{1} & \cdots & v_{n}
\end{array}\right| \neq 0 \text {. }
$$

We define $a_{1, n+1}=\cdots=a_{n-1, n+1}=0$ and $a_{n, n+1}=1$. And we determine $\left(a_{n 1}, \cdots\right.$, $\left.a_{n n}\right)$ by the system

$$
\left(\begin{array}{ccc}
a_{11} & \cdots & a_{1 n} \\
\cdots & \cdots & \cdots \\
a_{n-1,1} & \cdots & a_{n-1, n} \\
v_{1} & \cdots & v_{n}
\end{array}\right)\left(\begin{array}{c}
a_{n 1} \\
\vdots \\
\vdots \\
a_{n n}
\end{array}\right)=\left(\begin{array}{c}
0 \\
\vdots \\
0 \\
-v_{n+1}
\end{array}\right) .
$$

Then $\left(a_{n 1}, \cdots, a_{n, n+1}\right)$ is orthogonal to $\left(v_{1}, \cdots, v_{n+1}\right)$ and to $\left(a_{i 1}, \cdots, a_{t, n+1}\right)$, $i=1, \cdots, n-1$. Naturally $\left(a_{n 1}, \cdots, a_{n, n+1}\right) \in\left[C^{\omega}\right]^{n}$ and it does not vanish. The lemma is proved.

Q.E.D.

LeMmA 5.3. Let $\left(v_{1}, \cdots, v_{n}\right) \in\left[C^{\omega}(U)\right]^{n}$, and let $\left(v_{1}, \cdots, v_{n}\right) \neq O$ in $U$, 
where $U$ is a neighborhood of $O$ in $\boldsymbol{R}^{n}$. Then there are another neighborhood $U^{\prime}$ of $O$ and a vector function $\left(u_{1}, \cdots, u_{n-1}\right) \in\left[C^{\omega}\left(U^{\prime}\right)\right]^{n-1}$ such that $\sum_{k=1}^{n} \partial_{x_{k}} u_{i} \cdot v_{k}=0, i=1, \cdots, n-1$, and $\nabla u_{i} \cdot \nabla u_{j}=\delta_{i j}$ in $U^{\prime}$.

PROOF. If $v_{1}=\cdots=v_{n-1}=0$ particularly, it is enough to take $u_{i}=x_{i}$.

We consider the general case. By Lemma 5.2 there are $n-1$ unit vector functions $\left(a_{i 1}, \cdots, a_{i n}\right) \in\left[C^{\omega}\right]^{n}$ such that they are orthogonal to each other and so to $\left(v_{1}, \cdots, v_{n}\right)$. If we $\operatorname{set}\left(a_{n 1}, \cdots, a_{n n}\right)=\left(\sum_{k=1}^{n} v_{k}^{2}\right)^{-1 / 2}\left(v_{1}, \cdots, v_{n}\right)$, then it is in $\left[C^{\omega}\right]^{n}$ and the matrix $\left(a_{i j}\right)_{i, j=1}^{n}$ is orthogonal.

We write $e_{i}=\left(0, \cdots, \frac{i}{1}, 0, \cdots, 0\right)$ and define

$$
\boldsymbol{e}_{i}^{\prime}=\sum_{j=1}^{n} a_{i j} e_{j}, \quad i=1, \cdots, n
$$

Then they are unit vector functions which are orthogonal to each other, and we have

$$
\boldsymbol{e}_{i}=\sum_{j=1}^{n} a_{j i} \boldsymbol{e}_{j}^{\prime}
$$

Denoting by $\partial_{i}^{\prime}$ the differentiation to the direction $\boldsymbol{e}_{i}^{\prime}$, we have

$$
\begin{aligned}
\left(\partial_{i}^{\prime} f\right)(x) & =\lim _{h \rightarrow 0} h^{-1}\left[f\left(x+h e_{i}^{\prime}\right)-f(x)\right] \\
& =\sum_{j} a_{i j}\left(\partial_{x j} f\right)(x)
\end{aligned}
$$

so that

$$
\sum_{i} \partial_{i}^{\prime} f \cdot e_{i}^{\prime}=\sum_{i} \partial_{x_{i}} f \cdot e_{i}
$$

This implies that $\nabla f \cdot \nabla g$ is invariant by the above coordinate transformation. More precisely, writing $\nabla^{\prime} f=\left(\partial_{1}^{\prime} f, \cdots, \partial_{n}^{\prime} f\right)$, we have

$$
\nabla f \cdot \nabla g=\nabla^{\prime} f \cdot \nabla^{\prime} g \text {. }
$$

For any $x \in \boldsymbol{R}^{n}$ there are functions $\alpha_{i}(x), i=1, \cdots, n$, such that

$$
x=\sum_{i} x_{i} e_{i}=\sum_{i} \alpha_{i}(x) e_{i}^{\prime}
$$

Clearly $\alpha_{i}(x) \in C^{\omega}$. If we set $u_{j}(x)=\alpha_{j}(x)$, then

$$
u_{j}\left(x+h e_{i}^{\prime}\right)= \begin{cases}\alpha_{i}(x)+h & \text { if } j=i \\ \alpha_{j}(x) & \text { if } j \neq i,\end{cases}
$$

so that $\partial_{i}^{\prime} u_{j}=\delta_{i j}$. Therefore, for $1 \leqq i \leqq n-1$, 


$$
\sum_{k} \partial_{x_{k}} u_{i} \cdot v_{k}=\sum_{j}\left(\sum_{k} a_{j k} v_{k}\right) \partial_{j}^{\prime} u_{i}=\sum_{k} a_{i k} v_{k}=0
$$

Further from (5.3)

$$
\nabla u_{i} \cdot \nabla u_{j}=\nabla^{\prime} u_{i} \cdot \nabla^{\prime} u_{j}=\delta_{i j} \cdot
$$

From the above, $\left(u_{1}, \cdots, u_{n-1}\right)$ is the required vector function. Q.E.D.

\section{§6. Proof of Theorem 2.}

Let us fix any point on $S$ which may be assumed to be the origin. We consider our problem in the neighborhood of $O$. Thus we often omit the phrase "in a neighborhood of $O$ ". By Lemma 5.1 there is a function $\phi \in C^{\omega}$ with $|\nabla \phi|=1$ such that $\Omega$ and $\partial \Omega$ are expressed with $\phi>0$ and $\phi=0$ in a neighborhood of $O$, respectively. By Lemma 5.3 there is a vector function $\left(u_{1}, \cdots, u_{n-1}\right) \in\left[C^{\omega}\right]^{n-1}$ such that $u_{i}(O)=0, \nabla u_{i} \cdot \nabla \phi=0$ and $\nabla u_{i} \cdot \nabla u_{j}=\delta_{i j}$.

For the above functions $\phi, u_{1}, \cdots, u_{n-1}$, we consider the following $C^{\omega}$ mapping

$$
\Psi_{1}:\left\{\begin{array}{c}
\xi_{1}=u_{1} \\
\cdots \\
\xi_{n-1}=u_{n-1} \\
\xi_{n}=\phi .
\end{array}\right.
$$

It is clear that $\Psi_{1}$ is a one-to-one $C^{\omega}$ mapping from a neighborhood of $O$ onto another one. The inverse $\Psi_{1}^{-1}$ is also of class $C^{\omega}$ and the Jacobian of $\Psi_{1}$ is \pm 1 . Let us write $\xi=\left(\xi_{1}, \cdots, \xi_{n}\right)$ and $\xi^{\prime}=\left(\xi_{1}, \cdots, \xi_{n-1}\right)$. The image of $S$ lies on $\xi_{n}=0$, so that $S$ is an $(n-2)$-dimensional $C^{\infty}$ manifold in the $\xi^{\prime}$-space. In a neighborhood of $O$ the $\xi^{\prime}$-space is divided into two parts and $\partial_{1} \Omega$ is mapped into either of them.

Let us use again Lemmas 5.1 and 5.3, by replacing $n$ with $n-1$. Then there is a function $\psi\left(\xi^{\prime}\right) \in C^{\omega}$ with $\left|\nabla_{\xi^{\prime}} \psi\right|=1$ such that $S$ and the image $\Psi_{1}\left(\partial_{1} \Omega\right)$ are expressed with $\psi\left(\xi^{\prime}\right)=0$ and $\psi\left(\xi^{\prime}\right)>0$, respectively. Further there are functions $v_{1}\left(\xi^{\prime}\right), \cdots, v_{n-2}\left(\xi^{\prime}\right) \in C^{\omega}$ such that $v_{i}(O)=0, \nabla v_{i} \cdot \nabla \psi=0$ and $\nabla v_{i} \cdot \nabla v_{j}=\delta_{i j}$. For these functions we consider the following $C^{\omega}$ mapping

$$
\begin{aligned}
& \eta_{1}=v_{1}\left(\xi^{\prime}\right) \\
\cdots \cdots & \\
\Psi_{2}: \quad & \eta_{n-2}=v_{n-2}\left(\xi^{\prime}\right) \\
& \eta_{n-1}=\psi\left(\xi^{\prime}\right) \\
& \eta_{n}=\xi_{n} .
\end{aligned}
$$


The composite mapping of $\Psi_{1}$ and $\Psi_{2}$ is written as follows:

$$
\Psi_{2} \circ \Psi_{1}:\left\{\begin{array}{c}
\eta_{1}=w_{1}(x) \\
\cdots \cdots \\
\eta_{n}=w_{n}(x) .
\end{array}\right.
$$

Then $\Psi_{2} \circ \Psi_{1}$ is a $C^{\omega}$ one-to-one mapping from a neighborhood of $O$ onto another one. The inverse $\left(\Psi_{2} \circ \Psi_{1}\right)^{-1}$ is also of class $C^{\omega}$ and $w_{i}(O)=0$, $\nabla w_{i} \cdot \nabla w_{j}=\delta_{i j}$. Further $\Omega, \partial \Omega, \partial_{1} \Omega$ and $S$ are mapped into $\left\{\eta_{n}>0\right\},\left\{\eta_{n}=0\right\}$, $\left\{\eta_{n}=0, \eta_{n-1}>0\right\}$ and $\left\{\eta_{n}=\eta_{n-1}=0\right\}$, respectively. The Jacobian of $\Psi_{2}^{\circ} \circ \Psi_{1}$ is \pm 1 . For any two functions $f$ and $g$ we have

$$
\nabla_{\eta} f \cdot \nabla_{\eta} g=\nabla_{x} f \cdot \nabla_{x} g .
$$

Let $\rho(x)$ and $\phi(x)$ be the functions in Lemma 1.3 and Theorem 1, respectively. Then it is easily seen that

$$
c \rho(\eta) \leqq \phi(x) \leqq c^{-1} \rho(\eta), \quad c>0,
$$

in a neighborhood of $O$.

Hereafter let us regard the $\eta$-space newly as the $x$-space. And let us write $\left(\eta_{1}, \cdots, \eta_{n}\right)$ with $\left(x_{1}, \cdots, x_{n}\right)$. We define $\Sigma_{A}=\left\{x \in R^{n} ;|x|<A, x_{n}>0\right\}$. Let $R$ be any fixed sufficiently small positive number, and let $R^{\prime}$ be any fixed number with $0<R^{\prime}<R$. We write $\Sigma=\Sigma_{R}$ and $\Sigma^{\prime}=\Sigma_{R^{\prime}}$.

By (1.1) the function space $C_{(0)}^{1}(\bar{\Sigma})$ is defined. We denote by $V(\Sigma)$ the completion of $C_{(0)}^{1}(\bar{\Sigma})$ with the norm in $W^{1, p}(\Sigma)$. Similarly $V\left(\Sigma^{\prime}\right)$ is defined. The inner product in $L^{2}(\Sigma)$ is denoted by $(,)_{\Sigma}$. We remember that the Jacobian of $\Psi_{2} \circ \Psi_{1}$ is \pm 1 and (6.1) is valid, so that the solution of (0.3) satisfies

$$
\left(|\nabla u|^{p-2} \nabla u, \nabla v\right)_{\Sigma}+\left(|u|^{\alpha} u, v\right)_{\Sigma}=(f, v)_{\Sigma}, \quad v \in V\left(\Sigma^{\prime}\right),
$$

where $f \in W^{1, p^{*}}(\Sigma)$ from the assumption of Theorem 2.

Proof of Theorem 2. Let us take a function $\psi(x) \in C_{0}^{\infty}(\{|x|<R\})$ such that $\psi=1$ in $\left\{|x|<\left(R+R^{\prime}\right) / 2\right\}$. In (6.2) we can replace $u$ with $\psi u$, so that we can assume that $u \in V(\Sigma)$ and $u=0$ near $|x|=R$ without loss of generality. Let $S_{h}, T_{h}, P_{h}$ and $Q_{h}$ be the operators in Section 4 . Let $\gamma$ be a real number with $0<\gamma<1$, which may be chosen so close to 1 . Later we see that $\gamma$ depends on the number $\beta_{0}$ in Theorem 1 . Let the $y$-variable be connected with the $x$-variable by the mapping $\Phi_{h}$ defined in Section 4.

Hereafter let $w=Q_{h} u$. Obviously $T_{h} u=0$, if $y_{n}=0$ and $y_{n-1}>0$. And $\partial T_{h} u \in L^{p}(\Sigma)$ by Theorem 1 and (4.5). Hence we see that $w \in V(\Sigma)$ similarly 
to the proof of Lemma 1.2. We note that $w=0$ near $|x|=R$. Let $R^{\prime \prime}$ be a number with $0<R^{\prime \prime}<R^{\prime}$, and let us take a nonnegative function $\zeta \in C_{0}^{\infty}\left(\left\{|x|<R^{\prime \prime}\right\}\right)$. Clearly $S_{h} w=0$, if $x_{n}=0$ and $x_{n-1}>0$. From Theorem 1 there is a positive number " $\delta$ " such that $\tilde{\rho}^{-\delta} S_{h} \partial w \in L^{p}(\Sigma)$, so that $P_{h}\left(\zeta^{2} w\right) \in V\left(\Sigma^{\prime}\right)$ by Lemma 1.2 and (4.5).

For brevity we write $(,)_{\Sigma}$ simply with $($,$) . We have from (6.2)$

$$
\left(|\nabla u|^{p-2} \nabla u, \nabla P_{h}\left(\zeta^{2} w\right)\right)+\left(|u|^{\alpha} u, P_{h}\left(\zeta^{2} w\right)\right)=\left(f, P_{h}\left(\zeta^{2} w\right)\right) \text {. }
$$

First let us estimate the first term on the left-hand side of (6.3). We rewrite

$$
\begin{aligned}
& \left(|\nabla u|^{p-2} \nabla u, \nabla P_{h}\left(\zeta^{2} w\right)\right) \\
& =\left(|\nabla u|^{p-2} \nabla u, P_{h} \nabla\left(\zeta^{2} w\right)\right)+\left(|\nabla u|^{p-2} \nabla u, F_{h}\left(\nabla\left(\zeta^{2} w\right)\right)\right) .
\end{aligned}
$$

By (4.5) we see that

$$
\begin{aligned}
& \left|\left(|\nabla u|^{p-2} \nabla u, F_{k}\left(\nabla\left(\zeta^{2} w\right)\right)\right)\right| \\
& \leqq C\left[\int_{\Sigma} \tilde{\rho}^{1-1 / r}|\nabla u|^{p-1} \zeta(y)^{2}|(\nabla w)(y)| d x\right. \\
& \quad+\int_{\Sigma}\left|\left(\nabla \zeta^{2}\right)(y)\right||w(y)| \widetilde{\rho}^{1-1 / r}|\nabla u|^{p-1} d x \\
& \equiv I_{1}+I_{2}, \quad \text { say . }
\end{aligned}
$$

It follows from (4.2) and (4.5) that

$$
\begin{aligned}
I_{1} & \leqq C \int_{\Sigma} \tilde{\rho}^{1-1 / r} \zeta^{2}\left|T_{h} \nabla u\right|^{p-1}|\nabla w| d y \\
& \leqq C\left[\int_{\Sigma} \tilde{\rho}^{1-1 / \tau} \zeta^{2}\left|T_{h} \nabla u\right|^{p-1}\left|Q_{h} \nabla u\right| d y+\int_{\Sigma} \tilde{\rho}^{2(1-1 / r)} \zeta^{2}\left|T_{h} \nabla u\right|^{p} d y\right] .
\end{aligned}
$$

By Schwarz inequality

$$
\begin{aligned}
& \int_{\Sigma} \tilde{\rho}^{1-1 / r} \zeta^{2}\left|T_{h} \nabla u\right|^{p-1}\left|Q_{h} \nabla u\right| d y \\
& \leqq C\left(\int_{\Sigma} \zeta^{2} \widetilde{\rho}^{2(1-1 / r)}\left|T_{h} \nabla u\right|^{p} d y\right)^{1 / 2}\left(\int_{\Sigma} \zeta^{2}\left|T_{h} \nabla u\right|^{p-2}\left|Q_{h} \nabla u\right|^{2} d y\right)^{1 / 2} .
\end{aligned}
$$

Using Cauchy's inequality, we have for any $\varepsilon>0$

$$
\begin{aligned}
& \int_{\Sigma} \tilde{\rho}^{1-1 / r \zeta^{2}}\left|T_{h} \nabla u\right|^{p-1}\left|Q_{h} \nabla u\right| d y \\
& \leqq \varepsilon \int_{\Sigma} \zeta^{2}\left|T_{h} \nabla u\right|^{p-2}\left|Q_{h} \nabla u\right|^{2} d y+C \int_{\Sigma} \widetilde{\rho}^{2(1-1 / \tau)}\left|T_{h} \nabla u\right|^{p} d y,
\end{aligned}
$$

where the constant $C$ on the right-hand side depends on $\varepsilon$. Since 
$\tilde{\rho}^{2(1-1 / r)} \leqq C\left(x_{n-1}^{2}+x_{n}^{2}\right)^{1-1 / r}$ in $\Sigma$, we have the following inequality by returning to the original coordinate system:

$$
\begin{aligned}
& \int_{\Sigma} \tilde{\rho}^{2(1-1 / r)}\left|T_{h} \nabla u\right|^{p} d y \leqq C \int_{\Omega} \phi^{2(1-1 / r)}|\nabla u|^{p} d x \\
& \text { (by Theorem 1) } \leqq C\left(\|f\|_{p^{*}}\right)^{p^{*}} .
\end{aligned}
$$

From the above inequalities we obtain

$$
I_{1} \leqq \varepsilon \int_{\Sigma} \zeta^{2}\left|T_{h} \nabla u\right|^{p-2}\left|Q_{h} \nabla u\right|^{2} d y+\left(\|f\|_{p^{*}}\right)^{p^{*}}
$$

On the other hand by Hölder's inequality

$$
I_{2} \leqq C\left(\int_{\Sigma}\left|Q_{h} u\right|^{p} d y\right)^{1 / p}\left(\int_{\Sigma} \tilde{\rho}^{p^{*(1-1 / r)}}|\nabla u|^{p} d x\right)^{1 / p^{*}}
$$

Applying Lemma 4.2 and Theorem 1 to the right-hand side we have

$$
I_{2} \leqq C\left(\|f\|_{p^{*}}\right)^{p^{*}} \text {. }
$$

Combining (6.5), (6.6) with this inequality, we conclude that

$$
\begin{aligned}
& \left|\left(|\nabla u|^{p-2} \nabla u, F_{h}\left(\nabla\left(\zeta^{2} w\right)\right)\right)\right| \\
& \leqq \varepsilon \int_{\Sigma} \zeta^{2}\left|T_{h} \nabla u\right|^{p-2}\left|Q_{h} \nabla u\right|^{2} d y+C\left(\|f\|_{p^{*}}\right)^{p^{*}} .
\end{aligned}
$$

Next we estimate the first term on the right-hand side of (6.4). By Lemma 4.1

$$
\begin{aligned}
& \left(|\nabla u|^{p-2} \nabla u, P_{h} \nabla\left(\zeta^{2} w\right)\right) \\
& =\left(Q_{h}\left(|\nabla u|^{p-2} \nabla u\right), \nabla\left(\zeta^{2} w\right)\right)+\left(K_{h} T_{h}\left(|\nabla u|^{p-2} \nabla u\right), \nabla\left(\zeta^{2} w\right)\right) \\
& =\left(\zeta^{2} Q_{h}\left(|\nabla u|^{p-2} \nabla u\right), Q_{h} \nabla u\right)+\left(\zeta^{2} Q_{h}\left(|\nabla u|^{p-2} \nabla u\right), \nabla Q_{h} u-Q_{h} \nabla u\right) \\
& \quad+\left(Q_{h} u \cdot \nabla \zeta^{2}, Q_{h}\left(|\nabla u|^{p-2} \nabla u\right)\right)+\left(\zeta^{2} K_{h} T_{h}\left(|\nabla u|^{p-2} \nabla u\right), \nabla Q_{h} u\right) \\
& \quad+\left(K_{h} Q_{h} u \cdot \nabla \zeta^{2}, T_{h}\left(|\nabla u|^{p-2} \nabla u\right)\right) \\
& \equiv \sum_{i=1}^{s} J_{i}, \quad \text { say. }
\end{aligned}
$$

We have from (2.3)

$$
J_{1} \geqq c_{0} \int_{\Sigma} \zeta^{2}\left(\left|T_{h} \nabla u\right|^{p-2}+|\nabla u|^{p-2}\right)\left|Q_{h} \nabla u\right|^{2} d y
$$

By (2.5) and (4.5)

$$
J_{2} \leqq C \int_{\Sigma} \zeta^{2} \tilde{\rho}^{1-1 / \gamma}\left|T_{h} \nabla u\right|\left(\left|T_{h} \nabla u\right|^{p-2}+|\nabla u|^{p-2}\right)\left|Q_{h} \nabla u\right| d y
$$


Repeating the procedure in the proof of (6.6), we easily see that

$$
J_{2} \leqq \varepsilon \int_{\Sigma} \zeta^{2}\left(\left|T_{h} \nabla u\right|^{p-2}+|\nabla u|^{p-2}\right)\left|Q_{h} \nabla u\right|^{2} d y+C\left(\|f\|_{p^{*}}\right)^{p^{*}} .
$$

Similarly

$$
J_{3}, J_{4} \leqq \varepsilon \int_{\Sigma} \zeta^{2}\left(\left|T_{h} \nabla u\right|^{p-2}+|\nabla u|^{p-2}\right)\left|Q_{h} \nabla u\right|^{2} d y+C\left(\|f\|_{p^{*}}\right)^{p^{*}} .
$$

More easily we have

$$
J_{5} \leqq C\left(\|f\|_{p^{*}}\right)^{p^{*}} \text {. }
$$

From the above inequalities and (6.8) it follows that

$$
\begin{aligned}
& \left(c_{0}-3 \varepsilon\right) \int_{\Sigma} \zeta^{2}\left(\left|T_{h} \nabla u\right|^{p-2}+|\nabla u|^{p-2}\right)\left|Q_{h} \nabla u\right|^{2} d y \\
& \leqq\left(|\nabla u|^{p-2} \nabla u, P_{h} \nabla\left(\zeta^{2} w\right)\right)+C\left(\|f\|_{p^{*}}\right)^{p^{*}} .
\end{aligned}
$$

Now let us estimate the second term on the left-hand side of (6.3). From (0.5) and Lemma 4.1

$$
\begin{aligned}
& \left(|u|^{\alpha} u, P_{h}\left(\zeta^{2} w\right)\right) \\
& =\left(Q_{h}\left(|u|^{\alpha} u\right), \zeta^{2} Q_{h} u\right)+\left(K_{h} T_{h}\left(|u|^{\alpha} u\right), \zeta^{2} Q_{h} u\right) \\
& \geqq-C \int_{\Sigma} \zeta^{2} T_{h}\left(|u|^{1+\alpha}\right)\left|Q_{h} u\right| d y .
\end{aligned}
$$

By Hölder's inequality

$$
\int_{\Sigma} T_{h}\left(|u|^{1+\alpha}\right)\left|Q_{h} u\right| d y \leqq C\left(\int_{\Sigma}|u|^{(1+\alpha) p^{*}} d x\right)^{1 / p^{*}}\left(\int\left|Q_{h} u\right|^{p} d y\right)^{1 / p} .
$$

And by Sobolev's imbedding theorem

$$
\left(\int_{\Sigma}|u|^{(1+\alpha) p^{*}} d x\right)^{1 /(1+\alpha) p^{*}} \leqq C\left(\int_{\Sigma}|\nabla u|^{p} d x\right)^{1 / p},
$$

so that we have from (2.6) and Lemma 4.2

$$
\int_{\Sigma} T_{h}\left(|u|^{1+\alpha}\right)\left|Q_{h} u\right| d y \leqq C\left(\|f\|_{p^{*}}\right)^{(2+\alpha) /(p-1)} .
$$

Therefore it holds that

$$
\left(|u|^{\alpha} u, P_{h}\left(\zeta^{2} w\right)\right) \geqq-C\left(\|f\|_{p^{*}}\right)^{(2+\alpha) /(p-1)} .
$$

Lastly we estimate the right-hand side of (6.3). Repeating the above procedure, we see that 


$$
\begin{aligned}
\left(f, P_{h}\left(\zeta^{2} w\right)\right) & =\left(Q_{h} f, \zeta^{2} Q_{h} u\right)+\left(K_{h} T_{h} f, \zeta^{2} Q_{h} u\right) \\
& \leqq C\|f\|_{1, p^{*}}\left(\int_{\Sigma}|\nabla u|^{p} d x\right)^{1 / p} \\
& \leqq C\left(\|f\|_{1, p^{*}}\right)^{p^{*}}
\end{aligned}
$$

Let us combine (6.3), (6.4), (6.7), (6.9), (6.10) with this inequality. Further let us put $\varepsilon=c_{0} / 5$. Then it follows that

$$
\begin{aligned}
& \int_{\Sigma} \zeta^{2}\left(\left|T_{h} \nabla u\right|^{p-2}+|\nabla u|^{p-2}\right)\left|Q_{h} \nabla u\right|^{2} d y \\
& \leqq C\left[\left(\|f\|_{1, p^{*}}\right)^{p^{*}}+\left(\|f\|_{p^{*}}\right)^{(2+\alpha) /(p-1)}\right] .
\end{aligned}
$$

Clearly $\zeta(x)^{2} \leqq C\left(\zeta(y)^{2}+h^{2}\right)$ and $h\left|P_{h} \nabla u\right| \leqq\left|S_{h} \nabla u\right|+|\nabla u|$. In the same manner as the above we have thus

$$
h^{2} \int_{\Sigma}\left(\left|S_{h} \nabla u\right|^{p-2}+|\nabla u|^{p-2}\right)\left|P_{h} \nabla u\right|^{2} d x \leqq C\left(\|f\|_{p^{*}}\right)^{p^{*}}
$$

From this inequality and (6.11) we finally conclude that

$$
\int_{\Sigma} \zeta^{2}\left(\left|S_{h} \nabla u\right|^{p-2}+|\nabla u|^{p-2}\right)\left|P_{h} \nabla u\right|^{2} d x \leqq C A \text {, }
$$

where $C A$ is the right-hand side of (6.11).

By (2.5) we have

$$
\left.\left|P_{h}\right| \nabla u\right|^{p / 2}\left|\leqq C\left(\left|S_{h} \nabla u\right|^{(p-2) / 2}+|\nabla u|^{(p-2) / 2}\right)\right| P_{h} \nabla u \mid,
$$

so that it follows from (6.12) that

$$
\left.\left.\int_{\Sigma} \zeta^{2}\left|P_{h}\right| \nabla u\right|^{p / 2}\right|^{2} d x \leqq C A
$$

From this there are a function $v \in L^{2}(\Sigma)$ and a sequence $\left\{h_{\nu}\right\}$ with $h_{\nu} \rightarrow 0$ $(\nu \rightarrow \infty)$ such that

$$
\zeta P_{h_{\nu}}|\nabla u|^{p / 2} \rightarrow v \quad \text { in } L^{2}(\Sigma)
$$

By Lemma 4.1

$$
\begin{gathered}
\left(\zeta P_{h_{\nu}}|\nabla u|^{p / 2}, \varphi\right)=\left(|\nabla u|^{p / 2}, Q_{h}(\zeta \varphi)\right)+\left(K_{h_{\nu}} T_{h_{\nu}}(\zeta \varphi),|\nabla u|^{p / 2}\right), \\
\varphi \in C_{0}^{\infty}(\Sigma) .
\end{gathered}
$$

Since $K_{h} \rightarrow-\partial_{x_{n-1}} \tilde{\rho}$ as $h \rightarrow 0$, we have from Lemma 4.3 


$$
\begin{aligned}
& \left(\zeta P_{h_{2}}|\nabla u|^{p / 2}, \varphi\right) \\
& \rightarrow-\left(|\nabla u|^{p / 2}, \tilde{\rho} \partial_{x_{n-1}}(\zeta \varphi)\right)-\left(\partial_{x_{n-1}} \tilde{\rho} \cdot \zeta \varphi,|\nabla u|^{p / 2}\right) \\
& \quad=-\left(|\nabla u|^{p / 2}, \partial_{x_{n-1}}(\tilde{\rho} \zeta \varphi)\right) .
\end{aligned}
$$

Combining this with (6.14), we have $v=\zeta \tilde{\rho} \partial_{x_{n-1}}|\nabla u|^{p / 2}$. From (6.13) and (6.14) it holds that

$$
\int_{\Sigma} \zeta^{2} \widetilde{\rho}^{2}\left(\partial_{x_{n-1}}|\nabla u|^{p / 2}\right)^{2} d x \leqq C A
$$

Now we define for any function $v$

$$
\left\{\begin{array}{l}
v_{h}^{(i)}(x)=v\left(x_{1}, \cdots, x_{i-1}, x_{i}+h, x_{i+1}, \cdots, x_{n}\right) \\
\left(D_{h}^{(i)^{\prime}} v\right)(x)=h^{-1}\left(v_{h}^{(i)}(x)-v(x)\right) .
\end{array}\right.
$$

By replacing $P_{h}$ with $D_{h}^{(i)}$ for $1 \leqq i \leqq n-2$, we repeat the above procedure. Then we can obtain

$$
\int_{\Sigma} \zeta^{2}\left(\partial_{x_{i}}|\nabla u|^{p / 2}\right)^{2} d x \leqq C A
$$

Further we define for $\varepsilon>0$

$$
\kappa_{\varepsilon}(t)= \begin{cases}0 & \text { if } t<\varepsilon \\ t-\varepsilon & \text { if } t \geqq \varepsilon .\end{cases}
$$

In the above arguments we replace $P_{h}$ and $\zeta^{2}$ by $D_{h}^{(n)}$ and $\kappa_{s}\left(x_{n}\right)^{2} \zeta(x)^{2}$, respectively. Then we can obtain the following inequality in place of (6.15):

$$
\int_{\Sigma} \kappa_{\varepsilon}\left(x_{n}\right)^{2} \zeta^{2}\left(\partial_{x_{n}}|\nabla u|^{p / 2}\right)^{2} d x \leqq C A
$$

If we take $\varepsilon \rightarrow 0$, this inequality becomes

$$
\int_{\Sigma} x_{n}^{2} \zeta^{2}\left(\partial_{x_{n}}|\nabla u|^{p / 2}\right)^{2} d x \leqq C A
$$

Let $\theta \cdot \nabla$ be a $C^{1}$ vector field in $\bar{\Sigma}$, which is tangent to $\left\{x_{n}=0\right\}$. Writing $\theta=\left(\theta_{1}, \cdots, \theta_{n}\right)$, we have $\left|\theta_{n}(x)\right| \leqq C x_{n}$. From (6.15), (6.17) and (6.18) we obtain

$$
\int_{\Sigma} \zeta^{2} \widetilde{\rho}^{2}\left((\theta \cdot \nabla)|\nabla u|^{p / 2}\right)^{2} d x \leqq C A
$$

Let $1<s<2$. By Hölder's inequality 


$$
\begin{aligned}
& \left.\left.\int_{\Sigma} \zeta^{8}|(\theta \cdot \nabla)| \nabla u\right|^{p / 2}\right|^{s} d x \\
& \leqq\left(\int_{\Sigma} \tilde{\rho}^{-2 s /(2-s)} d x\right)^{(2-s) / 2}\left(\int_{\Sigma} \zeta^{2} \widetilde{\rho}^{2}\left((\theta \cdot \nabla)|\nabla u|^{p / 2}\right)^{2} d x\right)^{s / 2} .
\end{aligned}
$$

If $s$ is close to 1 , it holds that

$$
\int_{\Sigma} \tilde{\rho}^{-28 /(2-8)} d x<\infty \text {. }
$$

Indeed setting $t=s /(2-s)$ and $z=x_{n-1} / x_{n}^{\gamma}$, we have

$$
\int_{0}^{1} \frac{d x_{n-1}}{\left(x_{n-1}^{2}+x_{n}^{2 \gamma}\right)^{t}} \leqq x_{n}^{\gamma(1-2 t)} \int_{0}^{\infty} \frac{d z}{\left(z^{2}+1\right)^{t}}
$$

where we note that $\gamma<1$. Thus if $t$ is close to 1 , namely, $s$ is so, we get

$$
\int_{0}^{1} \int_{0}^{1} \frac{d x_{n-1} d x_{n}}{\left(x_{n-1}^{2}+x_{n}^{2 \gamma}\right)^{t}}<\infty
$$

which yields (6.21). Combining (6.19), (6.20) with (6.21), we finally conclude that

$$
\left.\left.\int_{\Sigma} \zeta|(\theta \cdot \nabla)| \nabla u\right|^{p / 2}\right|^{s} d x \leqq C A^{s / 2}
$$

More easily we can prove (6.22) in a neighborhood of each point of $\bar{\Omega}-S$. Let us put $\delta=s-1$. Returning to the original coordinate system and using (6.22), we complete the proof of Theorem 2 .

Q.E.D.

\section{§7. Proof of Theorem 3.}

Since $\left|\partial \phi^{1-\delta}\right| \leqq C \phi_{\phi^{-\delta}}$, by Theorem $1 \partial \phi^{1-\delta}|\nabla u|^{p-1} \in L^{p^{*}}(\Omega)$ for sufficiently small $\delta$. Thus it is enough to prove the inequality

$$
\left\|\phi^{1-\delta} \partial|\nabla u|^{p-1}\right\|_{p^{*}} \leqq C\left[\|f\|_{1, p^{*}}+\left(\|f\|_{p^{*}}\right)^{(1+\alpha) /(p-1)}+\left(\|f\|_{p^{*}}\right)^{(2+\alpha) / p}\right] \text {. }
$$

Proof of (7.1). Let $\zeta$ and $\Sigma$ be the same ones as in the proof of Theorem 2. We return to the procedure in the proof of Theorem 2 . Let $\rho=\rho(x)=\left(x_{n-1}^{2}+x_{n}^{2}\right)^{1 / 2}$. Since

$$
\left.\left|P_{h}\right| \nabla u\right|^{p-1}\left|\leqq C\left(S_{h}|\nabla u|^{p-2}+|\nabla u|^{p-2}\right)\right| P_{h} \nabla u \mid
$$

and $p^{*} / 2+(p-2) /(2(p-1))=1$, we have by Hölder's inequality

$$
\begin{aligned}
& \int_{\Sigma}\left(\left.\zeta \rho^{-\delta}\left|P_{h}\right| \nabla u\right|^{p-1} \mid\right)^{p^{*}} d x \leqq\left(\int_{\Sigma} \rho^{-2 p \delta /(p-2)}\left|S_{h} \nabla u\right|^{p} d x\right. \\
& \left.\quad+\int_{\Sigma} \rho^{-2 p \delta /(p-2)}|\nabla u|^{p} d x\right)^{(p-2) /(2(p-1))}\left(\int_{\Sigma} \zeta^{2}\left(\left|S_{h} \nabla u\right|^{p-2}+|\nabla u|^{p-2}\right)\left|P_{h} \nabla u\right|^{2} d x\right)^{p^{*} / 2} .
\end{aligned}
$$


Hence it follows from Theorem 1 and (6.12) that

$$
\begin{aligned}
& \int_{\Sigma}\left(\left.\zeta \rho^{-\delta}\left|P_{h}\right| \nabla u\right|^{p-1} \mid\right)^{)^{*}} d x \\
& \leqq C\left[\left(\|f\|_{1, p^{*}}\right)^{p^{*}}+\left(\|f\|_{p^{*}}\right)^{(2+\alpha) /(p-1)}\right],
\end{aligned}
$$

where we have used the inequality $\rho(y) \leqq C \rho(x)^{r}$. We write again with $C A$ the right-hand side of (7.2). Repeating the same argument as in the previous section and noting that $\rho \leqq C \tilde{\rho}$, we obtain from (7.2)

$$
\int_{\Sigma}\left(\left.\zeta \rho^{1-\delta}\left|\partial_{x_{n-1}}\right| \nabla u\right|^{p-1} \mid\right)^{p^{*}} d x \leqq C A .
$$

Next let $D_{h}^{(1)}$ be the operator in (6.16). Since from (2.5)

$$
\left.\left|D_{h}^{(s)}\right| \nabla u\right|^{p-1}\left|\leqq C\left(|\nabla u|^{p-2}+\left|\nabla u_{h}^{(s)}\right|^{p-2}\right)\right| \nabla D_{h}^{(s)} u \mid,
$$

we get the following inequality more easily than (7.2):

$$
\int_{\Sigma}\left(\left.\zeta\left|D_{h}^{(i)}\right| \nabla u\right|^{p-1} \mid\right)^{p^{*}} d x \leqq C A, \quad 1 \leqq i \leqq n-2,
$$

so that

$$
\int_{\Sigma}\left(\left.\zeta\left|\partial_{x_{i}}\right| \nabla u\right|^{p-1} \mid\right)^{p^{*}} d x \leqq C A, \quad i=1, \cdots, n-2 .
$$

We estimate finally the integral

$$
\int_{\Sigma}\left(\left.\zeta \rho^{1-\delta}\left|\partial_{x_{n}}\right| \nabla u\right|^{p-1} \mid\right)^{p^{*}} d x
$$

Let $D$ be a subdomain of $\Sigma$ with $\bar{D} \subset \Sigma$. First let us prove that

$$
\int_{D}\left(\left.|| \nabla u_{h}^{(n)}\right|^{p-2}-|\nabla u|^{p-2}|| D_{h}^{(n)} \partial_{x_{i}} u \mid\right)^{p *} d x \rightarrow 0, \quad \text { as } h \rightarrow 0, \quad 1 \leqq i \leqq n .
$$

Let $\kappa$ be a number such that $0<\kappa \leqq \min (p-2,1)$. We take $\kappa$ so that it is close to 0 , if necessary. We have from (2.5)

$$
\left.|| \nabla u_{h}^{(n)}\right|^{p-2}-\left.|\nabla u|^{p-2}\left|\leqq C\left(\left|\nabla u_{h}^{(n)}\right|^{p-2-\kappa}+|\nabla u|^{p-2-x}\right)\right| \nabla\left(u_{h}^{(n)}-u\right)\right|^{\boldsymbol{c}} .
$$

Let $q$ be a number such that $p^{*} / 2+\kappa p^{*} / p+1 / q=1$. By Hölder's inequality

$$
\begin{aligned}
& \int_{D}\left[\left(\left|\nabla u_{h}^{(n)}\right|^{p-2-\varepsilon}+|\nabla u|^{p-2-\kappa}\right)\left|\nabla\left(u_{h}^{(n)}-u\right)\right|^{\mid}\left|D_{h}^{(n)} \partial_{x_{i}} u\right|\right]^{p^{*}} d x \\
& \leqq C\left(\int_{D}\left|\nabla u_{h}^{(n)}\right|^{(p-2-2 \varepsilon) p^{*} / 2} d x+\int_{D}|\nabla u|^{(p-2-2 \varepsilon) p^{*} q / 2} d x\right)^{1 / q} \\
& \cdot\left(\int_{D}\left|\nabla\left(u_{h}^{(n)}-u\right)\right|^{p} d x\right)^{\kappa p^{*} / p}\left(\int_{D}\left(\left|\nabla u_{h}^{(n)}\right|^{p-2}+|\nabla u|^{p-2}\right)\left|D_{h}^{(n)} \partial_{x_{i}} u\right|^{2} d x\right)^{p * / 2} \text {. }
\end{aligned}
$$


Since $(p-2-2 \kappa) p^{*} q=2 p$, we have the following inequality by using (2.6) and (2.7):

$$
\begin{aligned}
& \int_{D}\left(\left.|| \nabla u_{h}^{(n)}\right|^{p-2}-|\nabla u|^{p-2}|| D_{h}^{(n)} \partial_{x_{i}} u \mid\right)^{p^{*}} d x \\
& \leqq C(f, D)\left(\int_{D}\left|\nabla\left(u_{h}^{(n)}-u\right)\right|^{p} d x\right)^{\kappa p^{* / p}},
\end{aligned}
$$

where the constant $C(f, D)$ depends on both $f$ and $D$. Therefore (7.6) has been proved.

Now we easily see that

$$
\begin{aligned}
& D_{h}^{(n)}\left(|\nabla u|^{p-2} \partial_{x_{n}} u\right)=\left|\nabla u_{h}^{(n)}\right|^{p-2} D_{h}^{(n)} \partial_{x_{n}} u \\
& \quad+(p-2) \partial_{x_{n}} u \cdot \int_{0}^{1}\left|t \nabla u_{h}^{(n)}+(1-t) \nabla u\right|^{p-4}\left(t \nabla u_{h}^{(n)}+(1-t) \nabla u\right) \cdot \nabla D_{h}^{(n)} u d t .
\end{aligned}
$$

We define $F_{h}$ as follows:

$$
D_{h}^{(n)}\left(|\nabla u|^{p-2} \partial_{x_{n}} u\right)=|\nabla u|^{p-2} D_{h}^{(n)} \partial_{x_{n}} u+(p-2) \partial_{x_{n}} u \cdot|\nabla u|^{p-4} \nabla u \cdot \nabla D_{h}^{(n)} u+F_{h} \cdot
$$

If we repeat the arguments in the proof of Proposition 2.2, similarly to (7.6) we see that $F_{h} \rightarrow 0$ in $L^{p^{*}}(D)$ as $h \rightarrow 0$. From the above equality,

$$
|\nabla u|^{p-2}\left|D_{h}^{(n)} \partial_{x_{n}} u\right| \leqq C\left(\left|D_{h}^{(n)}\left(|\nabla u|^{p-2} \partial_{x_{n}} u\right)\right|+|\nabla u|^{p-2} \sum_{i=1}^{n-1}\left|D_{h}^{(n)} \partial_{x_{i}} u\right|+\left|F_{h}\right|\right) .
$$

From this and (7.4) we have

$$
\begin{aligned}
& \int_{D}\left(\left.\zeta \rho^{1-\delta}\left|D_{h}^{(n)}\right| \nabla u\right|^{p-1} \mid\right)^{p^{*}} d x \leqq C\left[\int_{D}\left(\zeta \rho^{1-\delta}\left|D_{h}^{(n)}\left(|\nabla u|^{p-2} \partial_{x_{n}} u\right)\right|\right)^{p^{*}} d x\right. \\
& \quad+\sum_{i=1}^{n-1} \int_{D}\left(\zeta \rho^{1-\delta}|\nabla u|^{p-2}\left|D_{h}^{(n)} \partial_{x_{i}} u\right|\right)^{p^{*}} d x+\int_{D}\left(\zeta \rho^{1-\delta}\left|F_{h}\right|\right)^{p^{*}} d x \\
& \left.\quad+\int_{D}\left(\left.\zeta \rho^{1-\delta}|| \nabla u_{h}^{(n)}\right|^{p-2}-|\nabla u|^{p-2}|| D_{h}^{(n)} \nabla u \mid\right)^{p^{*}} d x\right] .
\end{aligned}
$$

Now from Proposition 2.2 there are two functions $v_{1}, v_{2} \in L_{10 \mathrm{c}}^{p^{*}}(D)$ such that

$$
\begin{aligned}
& |\nabla u|^{p-2} D_{h_{\nu}}^{(n-1)} \partial_{x_{n}} u \rightarrow v_{1}, \\
& |\nabla u|^{p-2} D_{h_{\nu}}^{(n)} \partial_{x_{n-1}} u \rightarrow v_{2} \quad \text { in } L^{p^{*}}(D) .
\end{aligned}
$$

And from (6.12) there is a function $w \in L^{p^{*}}(D)$ such that

$$
|\nabla u|^{p-2} P_{h_{\nu}} \partial_{x_{n}} u \rightarrow w \quad \text { in } L^{p^{*}}(D) \text {. }
$$

We prove that $\tilde{\rho} v_{1}=\tilde{\rho} v_{2}=w$ in $D$. Since $|\nabla u| \phi \in L^{p}(D)$ for any $\varphi \in C_{0}^{\infty}(D)$, it holds that 


$$
\left(P_{h_{\nu}} \partial_{x_{n}} u,|\nabla u|^{p-1} \varphi\right) \rightarrow(w,|\nabla u| \varphi)
$$

On the other hand

$$
\begin{aligned}
& \left(P_{h} \partial_{x_{n}} u,|\nabla u|^{p-1} \varphi\right) \\
& =\left(\partial_{x_{n}} P_{h} u,|\nabla u|^{p-1} \varphi\right)-\left(\partial_{x_{n}} \tilde{\rho} \cdot S_{h} \partial_{x_{n-1}} u,|\nabla u|^{p-1} \varphi\right) .
\end{aligned}
$$

Hence it follows from Lemma 4.3 and the remark continuing from Proposition 2.1 that

$$
\begin{aligned}
& \left(P_{h} \partial_{x_{n}} u,|\nabla u|^{p-1} \varphi\right) \\
& \quad \rightarrow-\left(\tilde{\rho} \partial_{x_{n-1}} u, \partial_{x_{n}}\left(|\nabla u|^{p-1} \varphi\right)\right)-\left(\partial_{x_{n}} \tilde{\rho} \cdot \partial_{x_{n-1}} u,|\nabla u|^{p-1} \varphi\right) \\
& \quad=-\left(\partial_{x_{n-1}} u, \partial_{x_{n}}\left(\tilde{\rho}|\nabla u|^{p-1} \varphi\right)\right) .
\end{aligned}
$$

Obviously

$$
\begin{aligned}
& \left(\tilde{\rho} D_{h}^{(n)} \partial_{x_{n-1}} u,|\nabla u|^{p-1} \varphi\right) \\
& \quad \rightarrow-\left(\partial_{x_{n-1}} u, \partial_{x_{n}}\left(\tilde{\rho}|\nabla u|^{p-1} \varphi\right)\right) .
\end{aligned}
$$

Therefore $|\nabla u| \tilde{\rho} v_{2}=|\nabla u| w$ in $D$, which implies that $\tilde{\rho}(x) v_{2}(x)=w(x)$ if $|\nabla u(x)| \neq 0$. Naturally $v_{2}(x)=w(x)=0$ if $|\nabla u(x)|=0$. Accordingly $\tilde{\rho} v_{2}=w$ in $D$. More easily we can prove that $v_{1}=v_{2}$ in $D$.

Let us denote $v_{1}$ and $v_{2}$ by $|\nabla u|^{p-2} \partial_{x_{n-1}} \partial_{x_{n}} u$. Then from the argument in the beginning of this section we have

$$
\int_{D}\left(\zeta \rho^{1-\delta}|\nabla u|^{p-2}\left|\partial_{x_{n-1}} \partial_{x_{n}} u\right|\right)^{p^{*}} d x \leqq C A
$$

More easily we see that

$$
\int_{D}\left(\zeta \rho^{1-\delta}|\nabla u|^{p-2}\left|\partial_{x_{i}} \partial_{x_{n}} u\right|\right)^{p *} d x \leqq C A,
$$

where $i \leqq n-2$. Hence it follows from (7.6) and (7.7) that

$$
\begin{aligned}
& \int_{D}\left(\left.\zeta \rho^{1-\delta}\left|\partial_{x_{n}}\right| \nabla u\right|^{p-1} \mid\right)^{p^{*}} d x \\
& \leqq C\left[A+\int_{D}\left(\zeta \rho^{1-\delta}\left|\partial_{x_{n}}\left(|\nabla u|^{p-2} \partial_{x_{n}} u\right)\right|^{p^{*}} d x\right] .\right.
\end{aligned}
$$

On the other hand from (6.2)

$$
\begin{aligned}
& \left|\partial_{x_{n}}\left(|\nabla u|^{p-2} \partial_{x_{n}} u\right)\right| \\
& \leqq C\left(|f|+|u|^{1+\alpha}+\sum_{i=1}^{n-1}\left|\partial_{x_{i}}\left(|\nabla u|^{p-2} \partial_{x_{i}} u\right)\right|\right) .
\end{aligned}
$$

Therefore 


$$
\int_{D}\left(\left.\zeta \rho^{1-\delta}\left|\partial_{x_{n}}\right| \nabla u\right|^{p-1} \mid\right)^{p^{*}} d x \leqq C\left[A+\left(\|u\|_{(1+\alpha) p^{*}}\right)^{(1+\alpha) p^{*}}\right] .
$$

By Sobolev's imbedding theorem and $(2.6), \quad\left(\|u\|_{(1+\alpha) p^{*}}\right)^{(1+\alpha) p^{*}} \leqq$ $C\left(\|f\|_{p^{*}}\right)^{(1+\alpha) p^{* /(p-1)}}$. Accordingly we finally obtain

$$
\int_{D}\left(\left.\zeta \rho^{1-\delta}\left|\partial_{x_{n}}\right| \nabla u\right|^{p-1} \mid\right)^{p^{*}} d x \leqq C\left[A+\left(\|f\|_{p^{*}}\right)^{(1+\alpha) p^{* /(p-1)}}\right] .
$$

By taking $D \rightarrow \Sigma$ and taking a partition of unity on $\bar{\Omega}$, we complete the proof of (7.1). Thus the proof of Theorem 3 is finished. Q.E.D.

\section{References}

[1] H. W. Alt and S. LuCKhaus, Quasilinear elliptic-parabolic differential equations, Math. Z., 183 (1983), 311-341.

[2] E. DiBenedetto, $C^{1+\alpha}$ local regularity of weak solutions of degenerate elliptic equations, Nonlinear Analysis, 7 (1983), 827-850.

[3] Z.-C. CHEN and C.-L. QIAN, Existence of generalized solution of degenerated quasilinear elliptic equation for the permeating problem, J. China Univ. Sci. Tech., vol. 12, no. 4 (1982), 8-16.

[4] K. HAYASIDA and H. NAGASE, On systems of variational inequalities with mixed boundary conditions, Funkcial. Ekvac., 28 (1985), 121-138.

[5] U. HoRNUNG, A parabolic-elliptic variational inequality, Manuscripta Math., 39 (1982), 155-172.

[6] G. N. JAkOLEv, Some properties of solutions of quasilinear elliptic equations, Proc. Steklov Inst. Math., 134 (1975), 441-458.

[7] J. LERAY and J. L. Lions, Quelques résultats de Visik sur les problèmes elliptiques non linéaires par les méthodes de Minty-Browder, Bull. Soc. Math. France, 93 (1965), 97-107.

[8] J. L. LEWIS, Regularity of the derivatives of solutions to certain degenerate elliptic equations, Indiana Univ. Math. J., 32 (1983), 847-858.

[9] G. M. Lieberman, Mixed boundary value problems for elliptic and parabolic differential equations of second order, J. Math. Anal. Appl., 113 (1986), 422-440.

[10] P. LINDQVIST, On the growth of the solutions of the differential equation $\operatorname{div}\left(|\nabla u|^{p-2} \nabla u\right)=0$ in n-dimensional space, J. Differential Equations, 58 (1985), 307-317.

[11] J. L. Lions, Quelques Méthodes de Résolution des Problèmes aux Limites Non Linéaires, Dunod/Gauthier Villars, 1969.

[12] V.E. MiersmanN, Zur gemischten Randwertaufgabe für die Minimalflächengleichung, Math. Nachr., 115 (1984), 125-136.

[13] M. K. V. MURThy and G. Stampacchia, A variational inequality with mixed boundary conditions, Israel J. Math., 13 (1972), 188-224.

[14] L. NiRenberg, Remarks on strongly elliptic partial differential equations, Comm. Pure Appl. Math., 8 (1955), 648-674.

[15] I. G. Petrovskiř, Partial Differential Equations, Gostefizdat, Moscow, 1955; Japanese Translation: Shōko-Shuppan, 1958.

[16] A.J. PRYDE, On an elliptic boundary value problem with mixed non-linear boundary conditions, Miniconference on Nonlinear Analysis, Canberra, 1984.

[17] E. Shamir, Regularization of mixed second order elliptic problems, Israel J. Math., 6 
(1968), 150-168.

[18] R. Teman, Applications de l'analyse convexe au calcul des variations, Lecture Notes in Math., 543, Springer, 1975, 208-237.

[19] P. TolKsDoRF, Regularity for a more general class of quasilinear elliptic equation, J. Differential Equations, 51 (1984), 126-150.

[20] H. BeIRÃo DA VEIGA, On the $W^{2, p}$-regularity for solutions of mixed problem, J. Math. Pures Appl., 53 (1974), 279-290.

[21] M. I. VISHIK, Systems of quasilinear elliptic differential equations, Trudy Moskov. Mat. Obshch., 12 (1963), 125-184 (Russian).

Present Address:

Department of Mathematics

Faculty of Science

KANAZAWA UNIVERSITY

MARU-NO-UCHI, KanaZAWA 920

AND

INTELLIGENCE BUSINESS SCHOOL OF KANAZAWA

hosai, Kanazawa 920 